\title{
APPLICATIONS OF THE GENERALIZED SINGULAR-VALUE DECOMPOSITION METHOD ON THE EIGENPROBLEM USING THE INCOMPLETE BOUNDARY ELEMENT FORMULATION
}

\author{
S. R. Kuo, W. Yein and Y. C. Wu \\ Department of Harbor and River Engineering, National Taiwan Ocean University, 2 Pei-Ning Road, \\ Keelung, Taiwan 20201, Republic of China.E-mail: b0121@mail.ntou.edu.tw.
}

(Received 30 September 1999, and in final form 14 March 2000)

\begin{abstract}
In this paper, four incomplete boundary element formulations, including the real-part singular boundary element, the real-part hypersingular boundary element, the imaginary-part boundary element and the plane-wave element methods, are used to solve the free vibration problem. Among these incomplete boundary element formulations, the real-part singular and the hypersingular boundary elements are of the singular type and the other two are of the regular type. When the incomplete formulation is used, the spurious eigensolution may be encountered. An auxiliary system, whose boundary conditions are linearly independent of those of the original system, is required in the proposed method in order to eliminate the spurious eigensolution. A mathematical proof is given to show that the spurious eigensolution will appear in both the original and auxiliary systems. As a result, one can eliminate spurious eigensolution by means of the generalized singular-value decomposition method. In addition to the spurious eigensolution problem, the regular boundary element formulation further suffers ill-conditioned behaviors in the problem solver. It is explained analytically using a circular domain why ill-conditioned behaviors exist. Two main ill-conditioned problems are the numerical instability of solution and the nonexistence of solution. To solve the numerical instability of solution problem existing in these proposed regular formulations, the Tikhonov's regularization technique is used to improve the condition number of the leading coefficient matrix; further, the generalized singular-value decomposition method is used to eliminate spurious eigensolutions. Numerical examples are given to verify the performance of these proposed methods, and the results match the analytical values well.
\end{abstract}

(C) 2000 Academic Press

\section{INTRODUCTION}

The Helmholtz equation is often encountered when one deals with the acoustic problem or the vibration problem. It is often used to describe the vibration of a structure [1], the acoustic cavity problem [2], the radiation of a wave [3] and the scattering of a wave [4]. To solve the Helmholtz equation numerically, usually, the finite element method (FEM) or the boundary element method (BEM) is adopted. The basic idea of the boundary element method is to use the so-called fundamental solution, which physically means the response at the observation point due to the action at the source point, and the Maxwell-Betti reciprocity theorem. (Some researchers call it the Green's identity in the potential problem.) However, for the Helmholtz operator, the complex-valued fundamental solution is required theoretically, and the boundary element formulation has been studied using such an 
approach [5, 6]. De Mey [7] later proposed a simplified formulation that used the real-part function of the complex-valued fundamental solution to construct the real-part BEM for the Helmholtz equation. This simplified method can save computation time since only the real-value computation is required. However, he only searched for the first eigenvalue, so the accompanying spurious eigenvalue phenomenon occurring due to use of the real-part formulation did not appear in his paper. Hutchinson [8] used the real-part BEM to solve vibration problems of a membrane, and he found that the spurious eigensolution phenomenon existed when he used this approach. He proposed a sorting technique to filter out the spurious eigenmode by examining the mode shape. Later, other real-valued formulations were developed, e.g., the multiple-reciprocity method (MR/BEM) [9-11] and the dual-reciprocity method (DR/BEM) [12-14]. In these algorithms, the Helmholtz equation is treated as a Poisson equation with an external source; therefore, the fundamental solution of the Laplace equation is considered. Kamiya et al. [15] found by means of a two-dimensional demonstration that the MR/BEM is nothing more than the real-part formulation. Subsequently, Yeih et al. [16] gave a general proof of this statement and found that the conventional MR/BEM and the real-part BEM formulation are not a complete solution method; as a result, a spurious eigensolution occurs. In order to filter out the spurious eigenvalue, several methods have been developed so far. Chen and Wong [17] first proposed combining the real-part singular and hypersingular boundary element equations and then checking the consistency of their corresponding modal shapes. Later, a threshold method was further proposed by Liou et al. [18]. Yeih et al. [19, 20] proposed the combined use of the real-part singular and hypersingular equations to construct an overdetermined system, and the singular-value decomposition method (SVD) was adopted to eliminate spurious eigensolutions. In the above-mentioned methods, the real-part singular and hypersingular methods are both used to filter out the spurious eigenvalue. Chang et al. [21] recently discovered that one can filter out the spurious eigenvalue within the real-part singular or hypersingular integral equation system. They used the domain partition technique and filtered out the spurious eigenvalue by simply adjusting the position of the internal links (the partition boundaries). However, in their methods, at least two meshes are required; thus, more computation is required. Nevertheless, it was then proved that one can successfully filter out the spurious eigenvalue using the real-part singular or hypersingular equation alone. This is quite meaningful from the computational point of view since the derivation of the hypersingular element requires a lot of theoretical derivation work, and numerical integration of a higher order shape function within the hypersingular element is usually not easy to accomplish. In the above-mentioned methods, the singular-type BEMs are used but they are all incomplete due to using the real-part formulation only. In addition to singular formulations, there exist other attempts using regular formulation and we will give a brief summary as follows.

De Mey [7] not only presented the real-part formulation in his paper, he also claimed that the imaginary-part formulation that used imaginary parts of kernels could be used in vibration problems. Notice that there exists no singular source in the imaginary-part formulations; therefore, this formulation does not need to treat the singular or even the hypersingular integral. Since no singular source exists in this formualtion, we call this formulation the regular formulation or source-free formulation. Further, it preserves the merit of the real-part formulation; that is, only the real-valued calculation is employed in such an approach. De Mey has also proposed using another source-free formulation in which a solution satisfying the Helmholtz equation is adopted as the auxiliary system. However, De Mey found that he could not reach the correct answer by means of this approach. From the mathematical point of view, no matter which kind of source-free formulation we use, the same situation shall we encounter. It is then puzzling why De Mey 
claimed that one could obtain the solution using one regular formulation but have no definite result using another regular formulation. Kim and Kang [22] used the wave-type base functions to analyze the free vibration of membranes. In this paper, the wave-type base functions, which are periodic along each element and propagating into the domain of interest, were selected to construct the needed equations. They pointed out that some incorrect answers would appear and they also explained this phenomenon as incompleteness of base functions. Later, Kang et al. [23] proposed another regular formulation using the so-called non-dimensional dynamic influence function. Simply speaking, their method took the response at any point inside the domain of interest as a linear combination of many non-singular point sources located on the selected boundary nodes. They claimed that their method worked very well and no numerical instability behaviors were reported. Recently, Chen et al. [24] used the circular domain and the property of circulants to examine theoretically the possibility of using the imaginary dual BEM as a solver for the Helmholtz eigenproblems. They reported that spurious eigensolutions also appeared in the imaginary dual BEM; however, no numerical examples were illustrated in their paper. Another non-singular boundary-type approach is the Trefftz method, which have been widely used to deal with many types of problems [25-27]. The boundary-type Trefftz method basically employs the complete set of solutions satisfying the governing equation as the beginning step. To derive the boundary integral equation, either the reciprocity law can be used, which is similar to that used in the conventional BEM, or the weight residual method can be used. A main benefit for the Trefftz method is that it does not involve singular integrals due to its solution basis functions ( $T$ functions); thus, it can be categorized into the regular boundary element method. A review article about the Trefftz method can be found in reference [28]. The above-mentioned boundary element formulations are all regular; however, they are not complete formulations as we will explain this later in this paper.

The main goal of this research is to seek for the solution method for the Helmholtz eigenproblem using an incomplete BEM formulation. For the incomplete singular BEM, the spurious eigensolutions are eliminated by means of the generalized singular-value decomposition method (GSVD). Unlike the traditional approaches, this method can successfully eliminate spurious eigensolution within the singular real integral equation or the hypersingular real integral equation. For the incomplete regular BEM, in addition to the spurious eigensolution numerical instability of solution may appear. The Tikhonov's regularization method is adopted with the GSVD, it can successfully solve the numerical instability of solution and possible spurious eigensolutions at the same time. Further, the limitation of using the incomplete regular BEM is studied analytically.

The present paper consists, apart from the literature review and the motivation of this study given in the introduction (section 1), of the following six sections. Section 2 describes the construction of various BEM formulations including the complex-valued formulation, real-singular formulation, real-hypersingular formulation, imaginary-regular formulations and plane-wave formulation for solving the Helmholtz equation. Further, the incomplete formulation is defined in the sense completeness of the basis functions and well-posedness of solution. In Section 3, the spurious eigensolution is re-examined based on the non-uniqueness of solution in the integral equation formulation, which results in the indefinite form of zero divided by zero. In section 4, a theorem to establish the theoretical background of this study is given. From this theorem, it is known that one can distinguish the true eigenvalue from the spurious one since spurious eigenvalues will exist in both the original problem and the auxiliary problem, where the boundary conditions of these two problems are linearly independent. Further, two alternative ways to filter out the spurious eigenvalues are proposed. In section 5, ill-conditioned behavior of regular BEM is examined 
by using a circular domain. It is shown that numerical instability of solution is a key issue for the regular BEM. Following that, the Tikhonov's regularization is introduced for stabilizing the problem. Furthermore, the non-existence of solution for proposed regular formulations for treating a multiply connected domain is theoretically examined. In section 6 , numerical examples are given to demonstrate the validity of the proposed methodology, and some important discussions are given. In the final section (section 7), some concluding remarks are made based on the results of this study.

\section{CONSTRUCTION OF VARIOUS BEM FORMULATIONS}

The problem we consider here is a two-dimensional Helmholtz equation written as

$$
\left(\nabla^{2}+k^{2}\right) u(\mathbf{x})=0, \quad \mathbf{x} \in \Omega
$$

where $u(\mathbf{x})$ is the potential (acoustic pressure in the acoustic problem or displacement function for the membrane vibration problem), $\mathbf{x}$ is the position vector of a point inside the domain of interest $\Omega, k$ is the wave number and $\nabla^{2}$ is the Laplacian operator. To solve this equation using the BEM, the complex-valued kernels are usually adopted, and the following equation can be derived [29]:

(singular integral equation: UT equation)

$$
c u(\mathbf{s})=R P V \int_{\Gamma} U_{C}(\mathbf{x}, \mathbf{s}) t(\mathbf{x}) \mathrm{d} \Gamma(\mathbf{x})-C P V \int_{\Gamma} T_{C}(\mathbf{x}, \mathbf{s}) u(\mathbf{x}) \mathrm{d} \Gamma(\mathbf{x}),
$$

(hypersingular integral equation: LM equation)

$$
c t(\mathbf{s})=C P V \int_{\Gamma} L_{C}(\mathbf{x}, \mathbf{s}) t(\mathbf{x}) \mathrm{d} \Gamma(\mathbf{x})-H P V \int_{\Gamma} M_{C}(\mathbf{x}, \mathbf{s}) u(\mathbf{x}) \mathrm{d} \Gamma(\mathbf{x}),
$$

where CPV, RPV and HPV denote the Cauchy principal value, the Riemann principal value and the Hadamard principal value, respectively; $t(\mathbf{s}) \equiv \partial u(\mathbf{s}) / \partial n_{S}$ with $n_{S}$ denotes the outnormal direction at point $\mathbf{s} ; \Gamma$ denotes the boundary enclosing the domain $\Omega$. It should be noted that the constant value of $c$ depends on where $\mathbf{s}$ is located. When $\mathbf{s}$ point approaches the smooth boundary, the value of $c$ is taken as $\pi$, and $c$ is equal to $2 \pi$ when $\mathbf{s}$ is located inside the domain $\Omega$. The four kernels used in the above equations are complex-valued and have the following properties: $\left(\nabla^{2}+k^{2}\right) U_{c}(\mathbf{x}, \mathbf{s})=2 \pi \delta(\mathbf{x}-\mathbf{s})$ where $\delta(\cdot)$ is the Dirac delta function and the $U_{C}(\mathbf{x}, \mathbf{s})$ satisfies the radiation condition, $T_{C}(\mathbf{x}, \mathbf{s})$ $\equiv \partial U_{C}(\mathbf{x}, \mathbf{s}) / \partial n_{x}, \quad L_{C}(\mathbf{x}, \mathbf{s}) \equiv \partial U_{C}(\mathbf{x}, \mathbf{s}) / \partial n_{S} \quad$ and $\quad M_{C}(\mathbf{x}, \mathbf{s}) \equiv \partial^{2} U_{C}(\mathbf{x}, \mathbf{s}) / \partial n_{x} \partial n_{S} . \quad$ Detailed expressions of these four kernels in the constant element scheme can be written as

$$
\begin{gathered}
U_{C}(\mathbf{x}, \mathbf{s})=-\frac{i \pi}{2} \mathrm{H}_{0}^{(1)}(k r), \\
T_{C}(\mathbf{x}, \mathbf{s})=-\frac{i k \pi}{2} \mathrm{H}_{1}^{(1)}(k r) \frac{y_{i} n_{i}}{r}, \\
L_{C}(\mathbf{x}, \mathbf{s})=\frac{i k \pi}{2} \mathrm{H}_{1}^{(1)}(k r) \frac{y_{i} \bar{n}_{i}}{r}, \\
M_{C}(\mathbf{x}, \mathbf{s})=-\frac{i k \pi}{2}\left\{-\frac{k}{r^{2}} \mathrm{H}_{2}^{(1)}(k r) y_{i} y_{j} n_{i} \bar{n}_{j}+\mathrm{H}_{1}^{(1)}(k r) \frac{n_{i} \bar{n}_{i}}{r}\right\},
\end{gathered}
$$


where $\mathrm{H}_{n}^{(1)}(k r)$ denotes the $n$th order Hankel function of the first kind; $r$ is the distance between the source point, $\mathbf{s}$, and the field point, $\mathbf{x} ; n_{i}$ is the $i$ th component of the outnormal vector at $\mathbf{x} ; \bar{n}_{i}$ is the $i$ th component of the outnormal vector at $\mathbf{s}$; and

$$
y_{i} \equiv x_{i}-s_{i}
$$

After constant element discretization is performed on the boundary, equations (2a) and (2b) can be written in the following matrix form:

(complex UT equation)

$$
[\overline{\mathbf{T}}-\mathbf{U}]\left[\begin{array}{l}
\mathbf{u} \\
\mathbf{t}
\end{array}\right]=[\mathbf{0}]
$$

(complex LM equation)

$$
[\mathbf{M}-\overline{\mathbf{L}}]\left[\begin{array}{l}
\mathbf{u} \\
\mathbf{t}
\end{array}\right]=[\mathbf{0}]
$$

where

$$
\begin{gathered}
U_{p q} \equiv \int_{\Gamma_{q}} U_{C}\left(\mathbf{x}, \mathbf{s}_{p}\right) \mathrm{d} \Gamma(\mathbf{x}), \\
\bar{T}_{p q} \equiv \pi \delta_{p q}-\int_{\Gamma_{q}} T_{C}\left(\mathbf{x}, \mathbf{s}_{p}\right) \mathrm{d} \Gamma(\mathbf{x}), \\
\bar{L}_{p q} \equiv \pi \delta_{p q}-\int_{\Gamma_{q}} L_{C}\left(\mathbf{x}, \mathbf{s}_{p}\right) \mathrm{d} \Gamma(\mathbf{x}), \\
M_{p q}=\int_{\Gamma_{q}} M_{C}\left(\mathbf{x}, \mathbf{s}_{p}\right) \mathrm{d} \Gamma(\mathbf{x}),
\end{gathered}
$$

in which $\Gamma_{q}$ represents the $q$ th element, $\mathbf{s}_{p}$ represents the collection point in the $p$ th element and $\delta_{p q}$ is the Kronecker delta symbol.

To avoid complicated computation in the complex-valued domain, the real-part BEM formulation is usually used to solve such an eigenproblem. The real-part BEM can be understood as using the real parts of the complex-valued kernels to replace the complex-valued ones in the above formulations. This means that one can simply use the following four kernels to replace complex-valued ones:

(real singular integral equation: real UT equation)

$$
c u(\mathbf{s})=R P V \int_{\Gamma} U_{R}(\mathbf{x}, \mathbf{s}) t(\mathbf{x}) \mathrm{d} \Gamma(\mathbf{x})-C P V \int_{\Gamma} T_{R}(\mathbf{x}, \mathbf{s}) u(\mathbf{x}) \mathrm{d} \Gamma(\mathbf{x})
$$

(real hypersingular integral equation: real LM equation)

$$
c t(\mathbf{s})=C P V \int_{\Gamma} L_{R}(\mathbf{x}, \mathbf{s}) t(\mathbf{x}) \mathrm{d} \Gamma(\mathbf{x})-H P V \int_{\Gamma} M_{R}(\mathbf{x}, \mathbf{s}) u(\mathbf{x}) \mathrm{d} \Gamma(\mathbf{x}),
$$


where

$$
\begin{gathered}
U_{R}(\mathbf{x}, \mathbf{s}) \equiv \operatorname{Real}\left(U_{C}(\mathbf{x}, \mathbf{s})\right) \\
T_{R}(\mathbf{x}, \mathbf{s}) \equiv \operatorname{Real}\left(T_{C}(\mathbf{x}, \mathbf{s})\right) \\
L_{R}(\mathbf{x}, \mathbf{s}) \equiv \operatorname{Real}\left(L_{C}(\mathbf{x}, \mathbf{s})\right) \\
M_{R}(\mathbf{x}, \mathbf{s}) \equiv \operatorname{Real}\left(M_{C}(\mathbf{x}, \mathbf{s})\right) .
\end{gathered}
$$

However, the real-part BEM formulations will result in the spurious eigensolution that has been mentioned in the Introduction. The existence of such a spurious eigenvalue has been explained by Yeih et al. [16] as being due to a lack of information contributed by the imaginary part of the complex-valued kernels.

Based on a similar idea, we can obtain the imaginary-part formulation, a regular formulation, by taking the imaginary part of equations (2a) and (2b) as follows:

(imaginary UT equation)

$$
0=\int_{\Gamma} U_{I}(\mathbf{x}, \mathbf{s}) t(\mathbf{x}) \mathrm{d} \Gamma(\mathbf{x})-\int_{\Gamma} T_{I}(\mathbf{x}, \mathbf{s}) u(\mathbf{x}) \mathrm{d} \Gamma(\mathbf{x})
$$

(imaginary LM equation)

$$
0=\int_{\Gamma} L_{I}(\mathbf{x}, \mathbf{s}) t(\mathbf{x}) \mathrm{d} \Gamma(\mathbf{x})-\int_{\Gamma} M_{I}(\mathbf{x}, \mathbf{s}) u(\mathbf{x}) \mathrm{d} \Gamma(\mathbf{x}),
$$

where

$$
\begin{gathered}
U_{I}(\mathbf{x}, \mathbf{s}) \equiv \operatorname{Imag}\left(U_{C}(\mathbf{x}, \mathbf{s})\right), \\
T_{I}(\mathbf{x}, \mathbf{s}) \equiv \operatorname{Imag}\left(T_{C}(\mathbf{x}, \mathbf{s})\right), \\
L_{I}(\mathbf{x}, \mathbf{s}) \equiv \operatorname{Imag}\left(L_{C}(\mathbf{x}, \mathbf{s})\right), \\
M_{I}(\mathbf{x}, \mathbf{s}) \equiv \operatorname{Imag}\left(M_{C}(\mathbf{x}, \mathbf{s})\right) .
\end{gathered}
$$

Notice that equations (9a) and (9b) can also be obtained from the Green's identity since $U_{I}(\mathbf{x}, \mathbf{s})$ and $L_{I}(\mathbf{x}, \mathbf{s})$ both are solutions of the Helmholtz equation. Although there is no singular source in the kernel functions, the kernels we use are still in a form of the radial distance, i.e., they are functions of $r$. To obtain the regular formulation, we should choose an auxiliary system which has several properties: (1) the solution of this auxiliary system can satisfy the Helmholtz equation; and (2) the solution of this auxiliary system needs to have the ability to construct enough equations. Using the imaginary-part equation is one alternative; another alternative is to use the fictitious boundary element method [30], in which the collocation points are still on the real boundary and the source densities are distributed on the fictitious boundary outside the physical domain. Here, we will suggest an alternative approach. Consider the solution of the auxiliary system as $\mathrm{e}^{\mathrm{i} k(m x+n y)}$, where $m^{2}+n^{2}=1$ and $(x, y)$ is the position vector of a specific source point; such a solution represents a plane wave. Let $x=\rho \cos \theta, y=\rho \sin \theta, m=\cos \phi$ and $n=\sin \phi$; then, we can rewrite the solution as $\mathrm{e}^{\mathrm{i} k \rho \cos (\theta-\phi)}$. In such an expression, the plane wave propagates in a direction that has an angle $\phi$ inclined with respect to the $x$-axis. Although this solution is not written as a two-point function, we can construct many equations by adjusting the angle 
$\phi$. To obtain the real-valued formulations, one can select $2 N$ directions of $\phi$ such that each plane-wave direction has an opposite plane wave propagating as shown in Figure 1. Then, we can choose the real-part of $\mathrm{e}^{\mathrm{i} k \rho \cos (\theta-\phi)}$ and imaginary part of $\mathrm{e}^{\mathrm{i} k \rho \cos (\theta-\phi)}$ as the basis since they both satisfy our requirements. Generally, this solution can be written as $P(\mathbf{x}, \phi)$. Plugging this solution into the Green's identity, we have

$$
0=\int_{\Gamma} P(\mathbf{x}, \phi) t(\mathbf{x}) \mathrm{d} \Gamma-\int_{\Gamma} \frac{\partial P(\mathbf{x}, \phi)}{\partial n_{x}} u(\mathbf{x}) \mathrm{d} \Gamma(\mathbf{x}) .
$$

No matter whether we solve the boundary unknowns using equations (9a), (9b) or (11), we do not encounter any singular or hypersingular integral; thus, the formulation qualifies as a regular BEM formulation. Of course, after the boundary quantities are all known, the internal field quantity cannot be represented by equations (9a), (9b) or (11) but it can be represented by the regular integral formulation shown in equation (2a) by setting $c$ equal to $2 \pi$. This inconsistency is due to that our formulations are of the direct type BEM. Kang's method [23] will not encounter such difficulties since their method is not of the direct BEM.

The above BEM formulations, no matter they are singular or regular, are incomplete. The meaning of incompleteness is defined as the lack of sufficient and well-posed constraint equations comparing to the complex-valued formulations, equations (2a) or (2b). For example, let us compare equations (2a) and (7a). The real-part singular integral equation although keeps singular property of the kernel, it loses the constraint equation from the imaginary part. On the contrary, the imaginary-part formulation (imaginary UT or LM equation) loses information from the real part of the complex-valued kernel. Plane-wave formulation can be proved to be equivalent to the Trefftz method for the simply connected

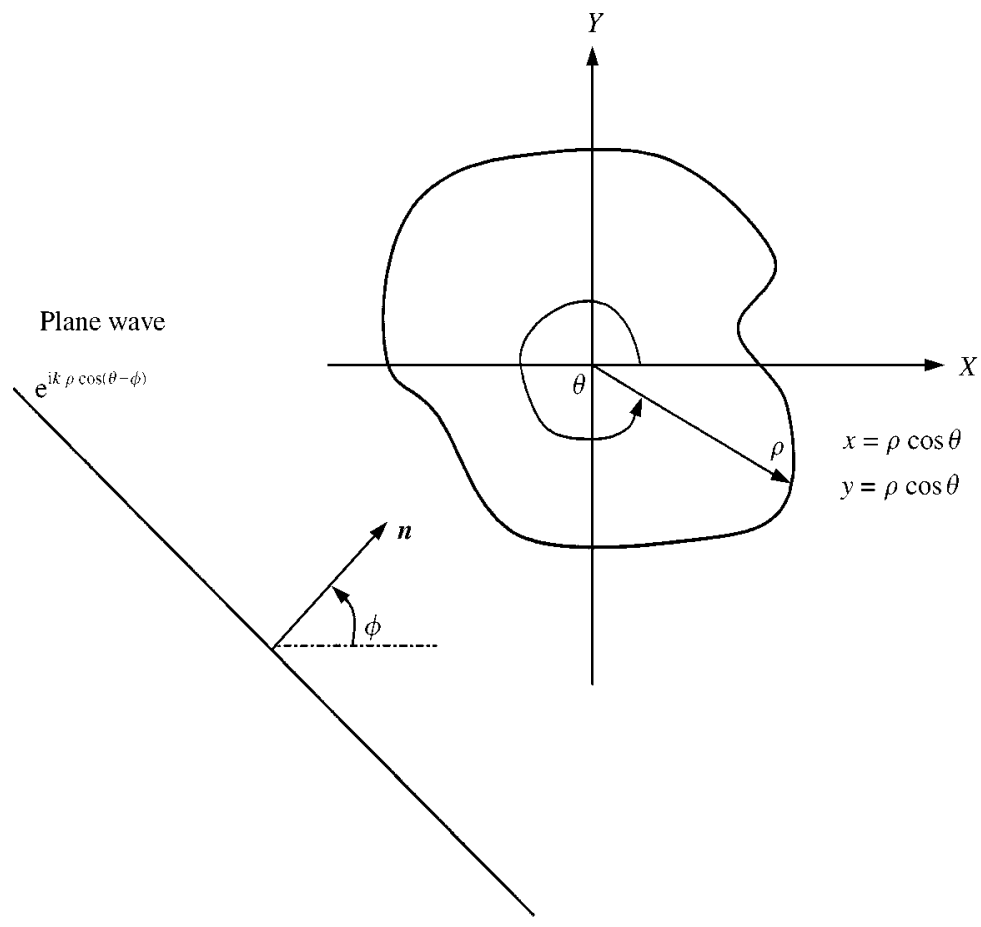

Figure 1. Plane-wave method. 
domain since they have the same basis functions, which will be illustrated later on. However, when a multiply connected domain is concerned, the complete set of the basis functions should include more functions. It means that the complete set of basis functions for the Trefftz method and plane-wave method must adjust according to the problem. Due to this property, we still categorize these methods as an incomplete BEM formulation.

Another reason for categorizing all methods proposed in this paper as incomplete formulations is that they all suffer some side-effects such as spurious eigensolution, numerical instability of solution or non-existence of solution. It is then very important for us to know how to deal with these side-effects.

\section{FILTERING OUT SPURIOUS EIGENSOLUTIONS}

In the following, we briefly review the conventional approach of filtering out spurious eigensolutions. First, let us rewrite equations (7a) or (7b) in the real-part BEM formulations and combine the set of boundary conditions in the following matrix form [31]:

(real UT equation)

$$
\left[\begin{array}{cc}
\overline{\mathbf{T}}_{R} & -\mathbf{U}_{R} \\
\boldsymbol{\alpha} & \boldsymbol{\beta}
\end{array}\right]\left[\begin{array}{l}
\mathbf{u} \\
\mathbf{t}
\end{array}\right]=[\mathbf{0}],
$$

(real LM equation)

$$
\left[\begin{array}{cc}
\mathbf{M}_{R} & -\overline{\mathbf{L}}_{R} \\
\boldsymbol{\alpha} & \boldsymbol{\beta}
\end{array}\right]\left[\begin{array}{l}
\mathbf{u} \\
\mathbf{t}
\end{array}\right]=[\mathbf{0}],
$$

where elements for the submatrices $\mathbf{U}_{R}, \overline{\mathbf{T}}_{R}, \overline{\mathbf{L}}_{R}$ and $\mathbf{M}_{R}$ are obtained by simply using the real parts of equations (6a), (6b), (6c) and (6d) respectively. It should be noted that $\boldsymbol{\alpha u}+\boldsymbol{\beta} \mathbf{t}=0$ in equations (12a) and (12b) is the homogeneous boundary condition, and that $\boldsymbol{\alpha}$ and $\boldsymbol{\beta}$ are diagonal matrices. For the eigenvalues, whether they are true or spurious, they cause rank deficiency of the leading coefficient matrix in equations (12a) or (12b); as a result; the inverse of the leading coefficient matrix does not exist. Nevertheless, previous studies [18-20] found that the spurious eigenvalues appearing in the real singular integral equation (real UT equation) will not cause rank deficiency in the real hypersingular integral equation (real LM equation) and vice versa. They further proposed creating an overdetermined system by combining the real-part UT and LM equations to filter out the spurious eigenvalues.

Let us take another look at the spurious eigenvalue problem beginning with results obtained for the Dirichlet and Neumann type problems for the unit circular cavity (see reference [32]) shown in Figures 2(a) and 2(b) using the real UT equation. In these figures, the minimum singular value is plotted versus the wave number since the singular-value decomposition method is widely recognized as the best method for representing the rank of a leading coefficient matrix $[33,34]$. From these figures, one can amazingly find that the spurious eigenvalues in both problems, one of the Dirichlet type and the other of the Neumann type, appear at the same wave numbers. Although there also exists a true eigenvalue $(k=3.83)$ which appears in both problems, the eigenmodes are $\mathrm{J}_{1}(3.83 \tau) \mathrm{e}^{ \pm \mathrm{i} \theta}$ and $\mathbf{J}_{0}^{\prime}(3.83 \tau)$ for the Dirichlet and Neumann problems respectively. In the above-mentioned terms, $\mathbf{J}_{n}(x)$ is the $n$th order Bessel function of the first kind and $\tau$ is the distance measuring from the center of the circle. Let us now examine the Dirichlet problem 


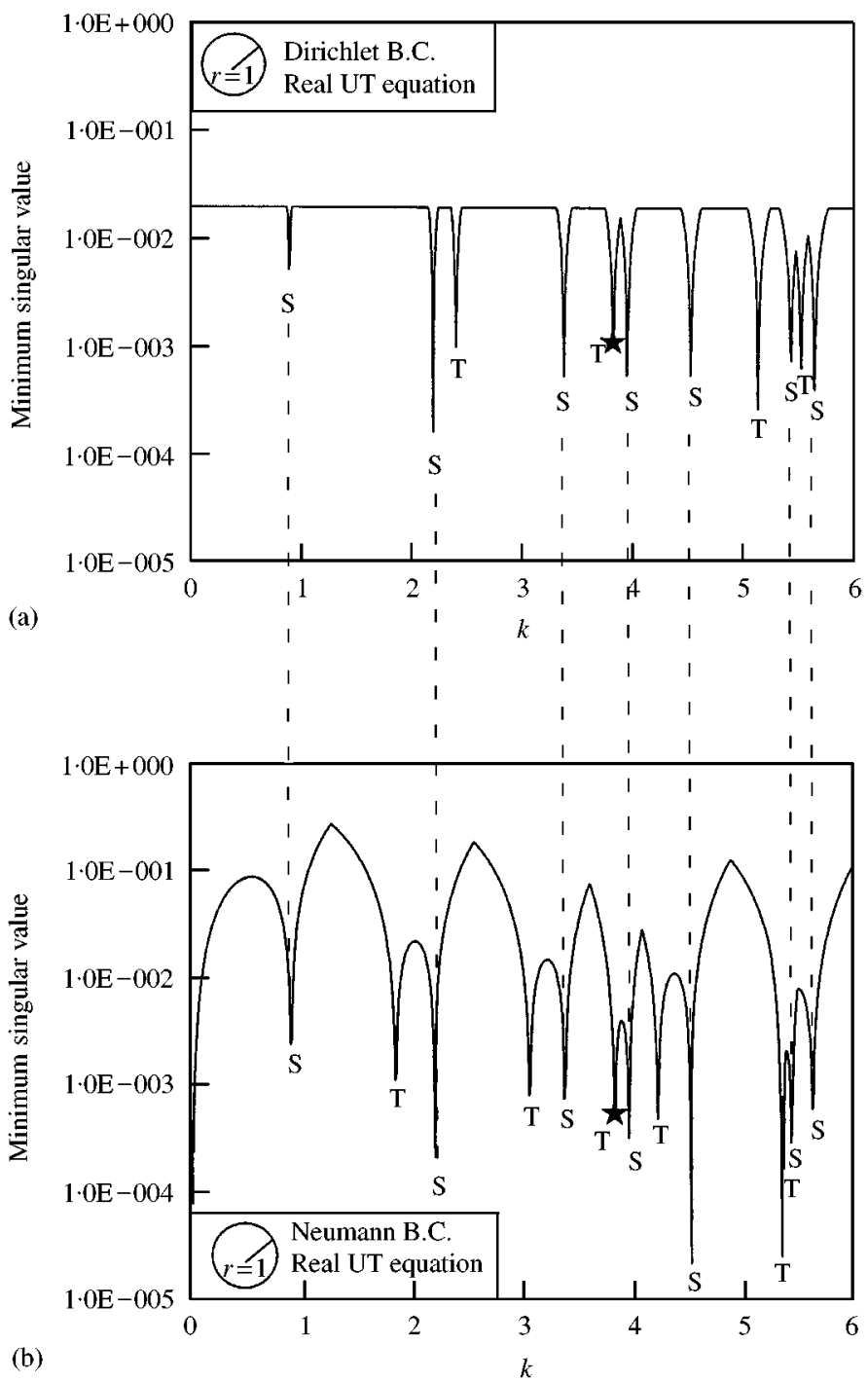

Figure 2. Eigenvalues for a unit circular domain using the real-part UT equation for the Dirichlet case (a) and the Neumann case (b). S: Spurious eigenvalue; T: True eigenvalue; $\star: 3 \cdot 83$.

first; the equation we obtain is actually

$$
\mathbf{U}_{R} \mathbf{t}=\overline{\mathbf{T}}_{R} \mathbf{u}=\mathbf{0}
$$

On the other hand, the equation for the Neumann problem is

$$
\overline{\mathbf{T}}_{R} \mathbf{u}=\mathbf{U}_{R} \mathbf{t}=\mathbf{0} .
$$

By comparing equations (13a) and (13b), and considering the conclusion we drew from Figures 2(a) and 2(b), we can say that the spurious eigenvalue, which causes rank deficiency for $\mathbf{U}_{R}$ and $\overline{\mathbf{T}}_{R}$ at the same time, may be a false alarm due to that degeneration of integral equation representation. Going back to equation (13a), let us assume that the following 
decomposition exists:

$$
\left(\mathbf{P} \boldsymbol{\Sigma}_{U} \mathbf{V}_{1}^{*}\right) \mathbf{t}=\left(\mathbf{P} \boldsymbol{\Sigma}_{T} \mathbf{V}_{2}^{*}\right) \mathbf{u}=\mathbf{0},
$$

where $\mathbf{P}$ is a non-singular matrix; $\boldsymbol{\Sigma}_{U}$ and $\boldsymbol{\Sigma}_{T}$ represent the diagonal matrices, which has values allocated on the diagonal line; $\mathbf{V}_{1}^{*}$ and $\mathbf{V}_{2}^{*}$ are the complex conjugate transpose of the unitary matrices $\mathbf{V}_{1}$ and $\mathbf{V}_{2}$. This decomposition method does exist and will be introduced in detail in the next section. Following that, we can obtain

$$
\mathbf{u}=\left(\mathbf{V}_{2} \boldsymbol{\Sigma}_{T}^{-1} \boldsymbol{\Sigma}_{U} \mathbf{V}_{1}^{*}\right) \mathbf{t}=\left(\mathbf{V}_{2} \boldsymbol{\Sigma} \mathbf{V}_{1}^{*}\right) \mathbf{t}=0
$$

provided that the inverse of the $\overline{\mathbf{T}}_{R}$ matrix is permissible, in which $\boldsymbol{\Sigma} \equiv \boldsymbol{\Sigma}_{T}^{-1} \boldsymbol{\Sigma}_{U}$.

From the numerical point of view, when the wave number approaches the true eigenvalue of the $\mathbf{U}_{R}$ matrix, the minimum diagonal value in $\boldsymbol{\Sigma}$ will be very near to zero which means rank deficiency exists. When the wave number approaches the spurious eigenvalue of the $\mathbf{U}_{R}$ matrix, the minimum diagonal value in the $\boldsymbol{\Sigma}_{U}$ matrix is very near to zero; as a result, it makes us to take this wave number as a true eigenvalue. However, the diagonal value in $\boldsymbol{\Sigma}$, which is obtained by dividing the minimum diagonal value in the $\boldsymbol{\Sigma}_{U}$ matrix by the corresponding entry in the $\boldsymbol{\Sigma}_{T}$ matrix, now is not near zero due to the cancelling effect. Analytically speaking, non-uniqueness of solution occurs at the spurious eigenvalues due to the undetermined form of zero divided by zero. Therefore, such an integral representation method theoretically will not lead to spurious eigenvalues if one can obtain the inverse operator analytically and the L'Hospital rule is adopted. Comparing the above-mentioned spurious eigenvalues with the fictitious resonance frequencies of the radiation problem for the exterior domain [35], one can conclude that such unreasonable rank deficiency problems occur due to the degeneration of the integral equations. Theoretically, they lead to the same mathematical problem of the undetermined form of zero divided by zero. A comprehensive review of solution non-uniqueness can be found in reference [36]. It should be noted that although only the real singular integral equation (real UT equation) has been used as an example to explain the phenomenon of spurious eigenvalues, the same mathematical structure exists in the real-part hypersingular integral equation (real-part LM equation). Further, one can conclude that the value at which the fictitious eigenvalue occurs is independent of the boundary conditions given but does depend on the integral equation one selects. The above statement sheds light on our goal of finding an algorithm to filter out the spurious eigenvalues within the real-part UT or LM equation itself; however, several questions must be answered first. First for all, the examples given above are Dirichlet- and Neumann-type problems. How can we generalize the cancel effect mentioned above to a Robin-type problem? Furthermore, how can one implement the above-mentioned cancel effect in the numerical sense? We will answer these questions one by one in the following sections.

\section{MATHEMATICAL BACKGROUND FOR THE GENERALIZED SINGULAR-VALUE DECOMPOSITION METHOD}

In this section, we will begin with a mathematical lemma.

Lemma 1. Given that the governing equation is a Helmholtz equation, $\left(\nabla^{2}+k^{2}\right) u(\mathbf{x})=0$, for a domain $\Omega$ enclosed by the boundary $\Gamma$ as shown in Figure 3, and that the overspecified homogeneous boundary conditions are $u(\mathbf{x})=0$ and $t(\mathbf{x})=0$ for $\mathbf{x}$ on the sub-boundary $\Gamma_{1} \subset \Gamma$, there exists a unique solution, $u(\mathbf{x})=0$ for $\mathbf{x} \in \Omega+\Gamma$. 


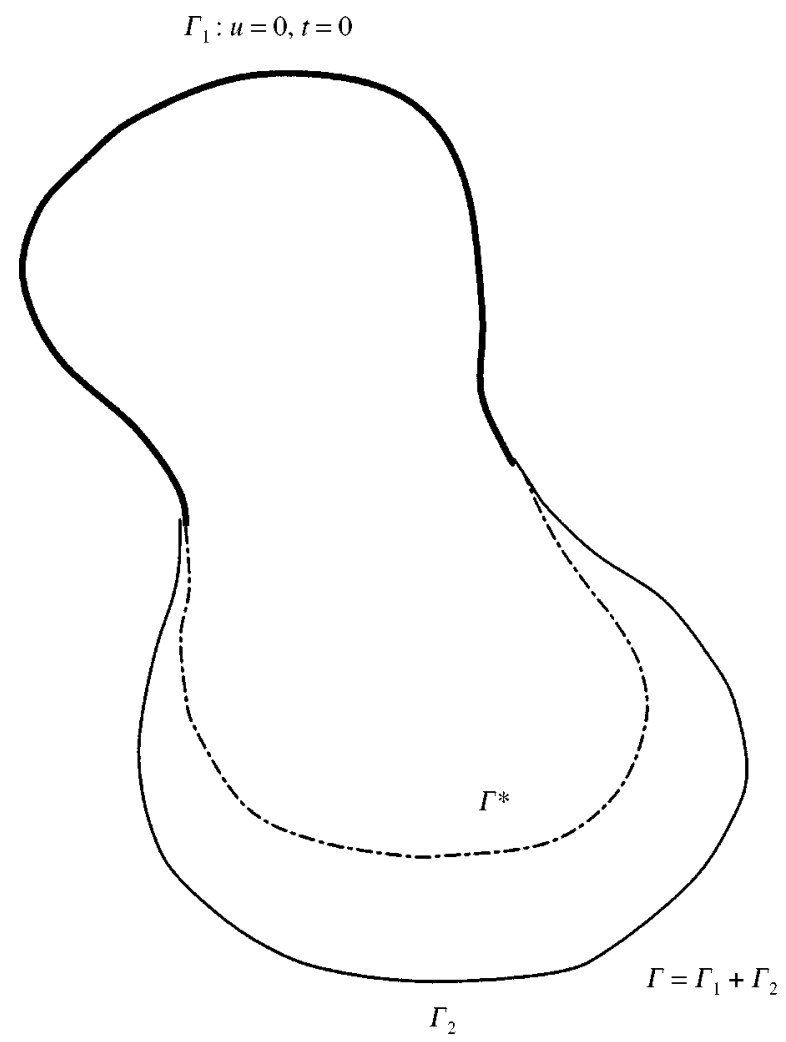

Figure 3. Illustration of a domain.

Proof. Assume there are two solution systems, $u^{(1)}(\mathbf{s})$ and $u^{(2)}(\mathbf{s})$, and both satisfy the Helmholtz equation in the domain $\Omega$. For the first solution set, assume that $u^{(1)}(\mathbf{x})=0$ and $t^{(1)}(\mathbf{x})=0$ for $\mathbf{x}$ on $\Gamma_{1}$. For the second solution set, assume that $u^{(2)}(\mathbf{x})=0$ but that $t^{(2)}(\mathbf{x}) \neq 0$ for $\mathbf{x}$ on $\Gamma_{2}$, where $\Gamma_{2}=\Gamma-\Gamma_{1}$. Then, from the Green's identity, one can obtain the following identity:

$$
\int_{\Gamma_{1}+\Gamma_{2}} t^{(2)}(\mathbf{x}) u^{(1)}(\mathbf{x}) \mathrm{d} \Gamma(\mathbf{x})=\int_{\Gamma_{1}+\Gamma_{2}} t^{(1)}(\mathbf{x}) u^{(2)}(\mathbf{x}) \mathrm{d} \Gamma(\mathbf{x}) .
$$

Based on the conditions given, equation (16) can be reduced to

$$
\int_{\Gamma_{2}} t^{(2)}(\mathbf{x}) u^{(1)}(\mathbf{x}) \mathrm{d} \Gamma(\mathbf{x})=0
$$

Since $t^{(2)}(\mathbf{x})$ is arbitrary for $\mathbf{x}$ on $\Gamma_{2}$, it can be concluded that $u^{(1)}(\mathbf{x})=0$ for $\mathbf{x}$ on $\Gamma_{2}$. We then have $u^{(1)}(\mathbf{x})=0$ for $\mathbf{x}$ on the whole boundary $\Gamma$.

Similarly, we can design another solution set, $u^{(3)}(\mathbf{s})$, which has $t^{(3)}(\mathbf{x})=0$ but $u^{(3)}(\mathbf{x}) \neq 0$ for $\mathbf{x}$ on $\Gamma_{2}$. Then, we will have the following identity:

$$
\int_{\Gamma_{2}} t^{(1)}(\mathbf{x}) u^{(3)}(\mathbf{x}) \mathrm{d} \Gamma(\mathbf{x})=0
$$


Finally, we have $t^{(1)}(\mathbf{x})=0$ for $\mathbf{x}$ on $\Gamma_{2}$; thus, we can conclude that $t^{(1)}(\mathbf{x})=0$ on the entire boundary.

Let us remember that the above derivation does not have any specific restriction of $\Gamma_{2}$. It means that if we choose any boundary $\Gamma^{*}$ such that $\Gamma_{1}+\Gamma^{*}$ constructs a closed boundary and encloses a subdomain $D$, we still have $u^{(1)}(\mathbf{x})=0$ and $t^{(1)}(\mathbf{x})=0$ for $\mathbf{x}$ on $\Gamma^{*}$. Therefore, we conclude that $u^{(1)}(\mathbf{x})=0$ and $t^{(1)}(\mathbf{x})=0$ for $\mathbf{x} \in \Gamma+\Omega$.

Before going further, we will first give a definition of the homogeneous linearly independent boundary conditions.

Definition. Two sets of boundary conditions, $\alpha_{1}(\mathbf{x}) u(\mathbf{x})+\beta_{1}(\mathbf{x}) t(\mathbf{x})=0$ and $\alpha_{2}(\mathbf{x}) u(\mathbf{x})+$ $\beta_{2}(\mathbf{x}) t(\mathbf{x})=0$, where $\boldsymbol{\alpha}_{1}(\mathbf{x}), \boldsymbol{\alpha}_{2}(\mathbf{x}), \boldsymbol{\beta}_{1}(\mathbf{x})$ and $\boldsymbol{\beta}_{2}(\mathbf{x})$ are given functions, are said to be homogeneous linearly independent boundary conditions if and only if

$$
\operatorname{det}\left\|\begin{array}{ll}
\alpha_{1}(\mathbf{x}) & \beta_{1}(\mathbf{x}) \\
\alpha_{2}(\mathbf{x}) & \beta_{2}(\mathbf{x})
\end{array}\right\| \neq 0
$$

for any $\mathbf{x}$ on the boundary.

According to Lemma 1, the following theorem can be proved.

Theorem 1. For the Helmholtz equation, given two systems having homogeneous linear independent boundary conditions on part of the boundary denoted as $\Gamma_{1}$, it is impossible for both systems to have the same eigensolution.

Proof. Assume that these two systems can have the same eigensolution, i.e., they have the same non-trivial eigenmode at the same eigenvalue. However, it is known that they have homogeneous linearly independent boundary conditions on the same boundary $\Gamma_{1}$. It can then be concluded that for their common eigenmode, $u(\mathbf{x})=0$ and $t(\mathbf{x})=0$ on the boundary $\Gamma_{1}$. From Lemma 1, the only possible eigenmode is $u(\mathbf{x})=0$; i.e., the trivial mode, which obviously leads to a contradiction. Therefore, we complete the proof.

Theorem 1 suggests that one can pick an auxiliary system whose boundary conditions are homogeneous linearly independent of those of the original system. Then, these two systems should not have the same eigensolution. Therefore, if one finds that at a specific wave number both systems have rank deficiency, then one can directly says that this wave number is not a true eigenvalue but a spurious eigenvalue.

It should be noted here that the minimum singular value versus wave number diagrams for the two systems should not be used to judge whether the eigenvalue is true or spurious. It is possible for two systems to have different eigensolutions at the same wave number owing to the existence of different eigenmodes.

We will propose two alternative ways to filter out spurious eigenvalues in the following:

Proposition 1. For the original problem, we symbolically write the equations we want to solve as

$$
[\mathbf{A}]\left[\begin{array}{l}
\mathbf{u} \\
\mathbf{t}
\end{array}\right]=\left[\begin{array}{cc}
\overline{\mathbf{T}}_{R} & -\mathbf{U}_{R} \\
\alpha_{1} & \beta_{1}
\end{array}\right]\left[\begin{array}{l}
\mathbf{u} \\
\mathbf{t}
\end{array}\right]=[\mathbf{0}] .
$$


Then, we choose another auxiliary system and symbolically express it as

$$
[\mathbf{B}]\left[\begin{array}{l}
\mathbf{u} \\
\mathbf{t}
\end{array}\right]=\left[\begin{array}{cc}
\overline{\mathbf{T}}_{R} & -\mathbf{U}_{R} \\
\alpha_{2} & \beta_{2}
\end{array}\right]\left[\begin{array}{l}
\mathbf{u} \\
\mathbf{t}
\end{array}\right]=[\mathbf{0}] .
$$

Consider the QR factorization of the matrix $\left[\begin{array}{l}\mathbf{A}^{\mathrm{T}} \\ \mathbf{B}^{\mathrm{T}}\end{array}\right]$; we have

$$
\left[\begin{array}{c}
\mathbf{A}^{\mathrm{T}} \\
\mathbf{B}^{\mathrm{T}}
\end{array}\right]=\left[\begin{array}{ll}
\mathbf{Q}_{11} & \mathbf{Q}_{12} \\
\mathbf{Q}_{21} & \mathbf{Q}_{22}
\end{array}\right]\left[\begin{array}{l}
\mathbf{R} \\
0
\end{array}\right]
$$

where

$$
\mathbf{Q}=\left[\begin{array}{ll}
\mathbf{Q}_{11} & \mathbf{Q}_{12} \\
\mathbf{Q}_{21} & \mathbf{Q}_{22}
\end{array}\right]
$$

is an orthogonal matrix and $\mathbf{R}$ is an upper triangular matrix.

Let

$$
\mathbf{Q}=\left[\begin{array}{ll}
\mathbf{Q}_{11} & \mathbf{Q}_{12} \\
\mathbf{Q}_{21} & \mathbf{Q}_{22}
\end{array}\right]=\left[\begin{array}{rr}
\mathbf{U} & 0 \\
0 & -\mathbf{V}
\end{array}\right]\left[\begin{array}{rr}
\mathbf{C} & \mathbf{S} \\
-\mathbf{S} & \mathbf{C}
\end{array}\right]\left[\begin{array}{ll}
\mathbf{W} & 0 \\
0 & \mathbf{Y}
\end{array}\right]^{\mathrm{T}}
$$

be the $\mathbf{C}$-S decomposition of $\mathbf{Q}$. Here, $\mathbf{U}, \mathbf{V}, \mathbf{W}$ and $\mathbf{Y}$ are orthogonal matrices, $\mathbf{C}=\operatorname{diag}\left(c_{i}\right)$ with $c_{i} \geqslant 0, \mathbf{S}=\operatorname{diag}\left(s_{i}\right)$ with $s_{i} \geqslant 0$ and $\mathbf{C}^{2}+\mathbf{S}^{2}=\mathbf{I}$. Then, we have

$$
\mathbf{A}=\mathbf{R}^{\mathrm{T}} \mathbf{Q}_{11}^{\mathrm{T}}=\left(\mathbf{R}^{\mathrm{T}} \mathbf{W}\right) \mathbf{C} \mathbf{U}^{\mathrm{T}}
$$

and

$$
\mathbf{B}=\mathbf{R}^{\mathrm{T}} \mathbf{Q}_{21}^{\mathrm{T}}=\left(\mathbf{R}^{\mathrm{T}} \mathbf{W}\right) \mathbf{S} \mathbf{V}^{\mathrm{T}} .
$$

The above method is called the generalized singular-value decomposition method [37]. Also, diagonal entries of the diagonal matrix $\mathbf{C}$ or $\mathbf{S}$, then, are called the generalized singular values. It can be found that due to the property $\mathbf{C}^{2}+\mathbf{S}^{2}=\mathbf{I}$, if the rank deficiency occurs only for matrix $\mathbf{A}$, then the minimum generalized singular value in matrix $\mathbf{C}$ will approach zero and vice versa. However, if rank deficiency occurs for both matrices $\mathbf{A}$ and $\mathbf{B}$, at least one diagonal entry in $\mathbf{R}$ will become zero, and the minimum generalized singular value will not approach zero theoretically. To examine equations (20) and (21) with equation (14), we can say that the generalized singular-value decomposition method can be used to extract the common part between two matrices as we expect in equation (14). Further, the common part, matrix $\mathbf{P}$ in equation (14), now is $\left(\mathbf{R}^{\mathrm{T}} \mathbf{W}\right)$ in equations (20) and (21). It is also important to note that if the common part in matrices $\mathbf{A}$ and $\mathbf{B}$ is a singular matrix, it should appear in matrix $\mathbf{R}$ but not in matrix $\mathbf{W}$ since matrix $\mathbf{W}$ is required to be orthogonal.

The above algorithm may encounter difficulties in numerical implementation. Since the indefinite form of zero divided by zero can be determined by the generalized singular-value decomposition in the theoretical sense, it is actually not so easy to implement in numerical sense once errors are introduced. In other words, it is not physically correct to implement an indefinite form of zero divided by zero in the numerical sense. A simple threshold generalized singular-value decomposition method is then proposed as follows. We known from the above statement that when the minimum value of diagonal entries of matrix $\mathbf{R}$ approaches zero, both matrices $\mathbf{A}$ and $\mathbf{B}$ face rank deficiency. This means the indefinite form of zero divided by zero is encountered. To avoid difficulty of numerical 
implementation for the indefinite form, one can simply skip such an attempt. Therefore, one can select a threshold value such that no numerical calculation is performed while the minimum diagonal value of matrix $\mathbf{R}$ is smaller than that threshold. Detailed discussions about this method will be given in section 6 later.

Proposition 2. From the above proposition, one can say that the spurious eigenvalue which cause both matrices $\mathbf{A}$ and $\mathbf{B}$ to degenerate must be embedded in matrix $\mathbf{R}$. We can check the rank of matrix $\left[\begin{array}{l}\mathbf{B}^{\mathrm{T}} \\ \mathbf{\mathbf { T } ^ { \mathrm { T } }}\end{array}\right]$ using the singular-value decomposition method or QR factorization [37]. In the singular-value decomposition method, if the minimum singular value approaches zero, then the rank is deficient. For $Q R$ factorization, if the matrix is rank deficient, then at least one diagonal entry in $\mathbf{R}$ is zero or very near zero. Therefore, comparing this information with the rank deficiency information obtained from the original problem, the spurious eigenvalue can then be easily eliminated.

It should be mentioned here that the matrix condensation is performed to reduce the dimensionality of the submatrices corresponding to the original and auxiliary systems before any QR factorization or GSVD operation. Therefore, the actual size of the matrices used in the numerical operation is half the size of that in equation (18).

\section{ILL-CONDITIONED BEHAVIORS OF THE REGULAR FORMULATION}

In the previous two sections, the spurious eigensolutions existing in the incomplete BEM formulations are eliminated by means of the generalized singular-value decomposition. Spurious eigensolutions do not only occur in the incomplete singular BEM formulations but also possibly appear in the regular formulation such as the imaginary UT or imaginary LM equation [24]. For regular formulation, the price we pay may not include spurious eigensolutions only. In order to understand what price we pay when the regular formulation is used, we will begin with the analytical derivation of the eigenvalue analysis for a circular domain with radius $\rho$. We will begin with imaginary formulations then the plane-wave method.

\subsection{IMAGINARY FORMULATION}

For simplicity, the imaginary UT formulation shown in equation (9a) is selected, and the boundary condition selected is of the Dirichlet type, i.e., $u=0$ on the boundary. A circular domain with radius of $\rho$ is selected as an example, the analytical derivation shown as follows is similar to previous studies $[38,39]$. First let us discretize the boundary into $2 N$ constant elements, we have the following matrix representation:

$$
\text { At }=\mathbf{0},
$$

where $\mathbf{t}$ is the flux value on the boundary and the matrix $\mathbf{A}$ is found to be symmetric and to have the circulant form as

$$
\mathbf{A}=\left[\begin{array}{ccccc}
a_{0} & a_{1} & a_{2} & \cdots & a_{2 N-1} \\
a_{2 N-1} & a_{0} & a_{1} & \cdots & a_{2 N-2} \\
a_{2 N-2} & a_{2 N-1} & a_{0} & \cdots & a_{2 N-3} \\
\vdots & \vdots & \vdots & \ddots & \vdots \\
a_{1} & a_{2} & a_{3} & \cdots & a_{0}
\end{array}\right]
$$


in which

$$
a_{n}=U_{I}(\mathbf{x}, \mathbf{s}) \cdot \rho \Delta \theta=U_{I}\left(\theta_{n}\right) \cdot \rho \Delta \theta, \Delta \theta \equiv \frac{2 \pi}{2 N}, \quad \theta_{n}=n \Delta \theta, n=0,1, \ldots, 2 N-1
$$

and $U_{I}(\mathbf{x}, \mathbf{s})=\operatorname{Imag}\left[U_{C}(\mathbf{x}, \mathbf{s})\right]=U_{I}\left(\theta_{n}\right)=\sum_{m=-\infty}^{\infty} \mathbf{J}_{m}(k \rho) \mathbf{J}_{m}(k \rho) \cos \left(m \theta_{n}\right)$. Notice that the kernel $U_{I}$ is a function of the position vectors of the source point, $\mathbf{s}$, and field point $\mathbf{x}$. However, when $\mathbf{x}$ and $\mathbf{s}$ are both located on the boundary of the circular domain with definite radius $\rho$, the kernel then becomes a function of the relative angle between two elements, that is a function of $\theta_{n}$.

From the definition, it is easy to verify that $a_{2 N-k}=a_{k}$. For a discretized Fourier's transformation matrix, $\mathfrak{R}$, that is

$$
\mathfrak{R}=\frac{1}{\sqrt{2 N}}\left[\begin{array}{ccccc}
1 & 1 & 1 & \cdots & 1 \\
\alpha_{0}^{1} & \alpha_{1}^{1} & \alpha_{2}^{1} & \cdots & \alpha_{2 N-1}^{1} \\
\alpha_{0}^{2} & \alpha_{1}^{2} & \alpha_{2}^{2} & \cdots & \alpha_{2 N-1}^{2} \\
\vdots & \vdots & \vdots & \cdots & \vdots \\
\alpha_{0}^{2 N-1} & \alpha_{1}^{2 N-1} & \alpha_{2}^{2 N-1} & \cdots & \alpha_{2 N-1}^{2 N-1}
\end{array}\right]
$$

where $\alpha_{1}=\mathrm{e}^{(2 \pi / 2 N) i}=\mathrm{e}^{\mathrm{i} \Delta \theta}$ and $\alpha_{m} \equiv\left(\alpha_{1}\right)^{m}$, we have

$$
\overline{\mathfrak{R}}^{T} \mathfrak{R}=\mathbf{I},
$$

where $\overline{\mathfrak{R}}$ is the complex conjugate of $\mathfrak{R}$. Further, we have

$$
\alpha_{m}^{2 N-n}=\alpha_{m}^{-n}, \quad \alpha_{2 N-m}=\bar{\alpha}_{m} \quad \text { and } \quad \alpha_{m}^{2 N}=1 .
$$

It can then be concluded that the matrix $\mathbf{A}$ can be decomposed into

$$
\mathbf{A} \mathfrak{R}=\mathfrak{R} \tilde{\mathbf{A}}
$$

where $\tilde{\mathbf{A}}$ is a diagonal matrix with diagonal entries

$$
\tilde{a}_{m}=\sum_{n=0}^{2 N-1} a_{n} \alpha_{m}^{n}=\sum_{n=0}^{2 N-1} a_{n}\left(\alpha_{m}^{n}+\alpha_{m}^{-n}\right) / 2+\sum_{n=0}^{2 N-1} a_{n}\left(\alpha_{m}^{n}-\alpha_{m}^{-n}\right) / 2
$$

and the last summation on the right-hand side of the above equation is zero since

$$
\sum_{n=0}^{2 N-1} a_{n}\left(\alpha_{m}^{n}-\alpha_{m}^{-n}\right) / 2=\sum_{n=0}^{2 N-1} a_{n}\left(\alpha_{m}^{n}-\alpha_{m}^{2 N-n}\right) / 2=\sum_{n=0}^{2 N-1}\left(a_{n} \alpha_{m}^{n} / 2-a_{2 N-n} \alpha_{m}^{2 N-n} / 2\right)=0 .
$$

It can then be concluded that

$$
\tilde{a}_{m}=\sum_{n=0}^{2 N-1} a_{n} \alpha_{m}^{n}=\sum_{n=0}^{2 N-1} a_{n}\left(\alpha_{m}^{n}+\alpha_{m}^{-n}\right) / 2=\sum_{n=0}^{2 N-1} a_{n} \cos (m n \Delta \theta), \quad m=0,1,2, \ldots, 2 N-1 .
$$


Notice that the following property holds:

$$
\begin{aligned}
\tilde{a}_{2 N-m} & =\sum_{n=0}^{2 N-1} a_{n} \cos [n(2 N-m) \Delta \theta]=\sum_{n=0}^{2 N-1} a_{n} \cos [2 n \pi-m n \Delta \theta] \\
& =\sum_{n=0}^{2 N-1} a_{n} \cos [n m \Delta \theta]=\tilde{a}_{m}
\end{aligned}
$$

for $m=1,2, \ldots, N-1$.

When $N$ becomes very big, it yields

$$
\begin{aligned}
\tilde{a}_{m} & \cong \rho \int_{0}^{2 \pi} U_{I}(\theta) \cos (m \theta) \mathrm{d} \theta=\rho \int_{0}^{2 \pi} \sum_{l=-\infty}^{\infty} \mathbf{J}_{l}(k \rho) \mathbf{J}_{l}(k \rho) \cos (l \theta) \cos (m \theta) \mathrm{d} \theta \\
& =2 \pi \rho \mathbf{J}_{m}(k \rho) \mathbf{J}_{m}(k \rho), \quad m=0,1,2, \ldots, N .
\end{aligned}
$$

Taking the determinant value of matrix $\mathbf{A}$, we have

$$
\begin{aligned}
\operatorname{det}(\mathbf{A}) & =\operatorname{det}(\tilde{\mathbf{A}}) \cong \widetilde{a_{0}} \widetilde{a_{N}} \prod_{m=1}^{N-1} \tilde{a}_{m}^{2} \\
& =(2 \pi \rho)^{2 N} \mathbf{J}_{0}^{2}(k \rho) \mathbf{J}_{N}^{2}(k \rho) \prod_{m=1}^{N-1}\left[\mathbf{J}_{m}^{2}(k \rho)\right]^{2} .
\end{aligned}
$$

In equation (32), we find that the expected rank deficiency of matrix A occurs at the wave number that makes $\mathbf{J}_{m}(k \rho)=0$ for the imaginary UT equation. Similar derivation [24] from the imaginary LM equation can be conducted, and we find that rank deficiency occurs at a wave number that makes $\mathbf{J}_{m}(k \rho)=0$ or $\mathbf{J}_{m}^{\prime}(k \rho)=0$, where $\bullet^{\prime}$ means to take derivative with respect to $\rho$, i.e., to take derivative with respect to the radial direction. Combining these derivations, we can conclude that $\mathrm{J}_{m}(k \rho)=0$ is the true eigenequation. In this case, the imaginary UT equation does not have spurious eigenvalues theoretically, but the imaginary LM equation may have spurious eigenvalues [24]. Although spurious eigensolutions are expected, we can combine both sets of equations together and then use the singular-value decomposition method to filter them out theoretically $[19,20]$. It seems that we should obtain the solution from the imaginary-part equations. Figure 4 shows the minimum singular-value plot obtained using the combination of imaginary UT and LM equations with the singular-value decomposition technique. From the figure, we can see that the true eigenvalue cannot be found. The reason is as follows. Let us examine equation (32); we find that there exists a very small value for $\mathbf{J}_{m}(k \rho)$ and/or $\mathbf{J}_{m}^{\prime}(k \rho)$ in the imaginary UT and LM equations when $m$ is big enough and $k \rho$ is small. Such a small value will mislead us to believe that the leading coefficient matrix is rank deficient in the numerical sense; furthermore, this phenomenon becomes worse when the number of elements increases. We can say that the high order modes contaminate the eigenvalue search. De Mey [7] claimed that he could find the first eigenvalue for the Dirichlet case using the imaginary UT method. Based on our arguments, this declaration is not generally true. Numerically speaking, the high order mode contamination is an ill-posed behavior that is often called numerical instability. Therefore, we find that the price we pay when we use the regular formulation is that the solution is not continuously dependent of data (numerical instability). When the solution is solvable, numerical instability is encountered.

How can we deal with this numerical instability phenomenon? Let us remind ourselves that the influence matrix we encounter here is an ill-conditioned matrix, so numerical 


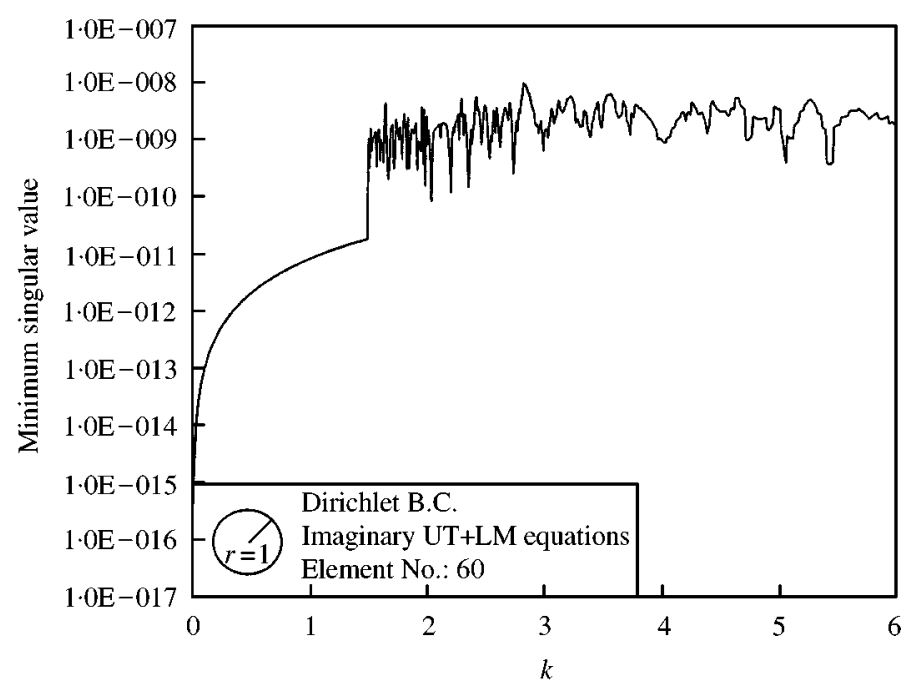

Figure 4. Contamination in eigenvalue searching using the imaginary BEM formulation.

instability occurs. In the case of the inverse problem, numerical instability is often encountered, and the Tikhonov's regularization method [40] is often used to improved the condition number of an ill-conditioned matrix. Here, we will briefly introduced the Tikhonov's regularization method. An ill-conditioned real matrix $\mathbf{C}$ has the singular-value decomposition

$$
\mathbf{C}=\mathbf{P} \Sigma \mathbf{V}^{\mathrm{T}}
$$

where $\mathbf{P}$ is an orthogonal matrix constructed by right singular vectors, $\Sigma$ is a diagonal matrix with singular values, $\sigma_{i}$, allocating in the diagonal line with $\sigma_{1} \leqslant \sigma_{2} \leqslant \cdots \leqslant \sigma_{\max }$ and $\mathbf{V}^{\mathrm{T}}$ is the transpose of an orthogonal matrix constructed by the left singular vectors. The condition number is defined as $\bar{C} \equiv \sigma_{\max } / \sigma_{1}$. When $\bar{C}$ is very big, the matrix $\mathbf{C}$ is said to be an ill-conditioned matrix, and the numerical instability is expected. The Tikhonov's regularization method basically provides a way to improve the condition number by adding a small quantity to the singular value.

That is, a new matrix $\widetilde{\mathbf{C}}$ is defined as

$$
\widetilde{\mathbf{C}} \equiv \mathbf{C}+\mathbf{P} \Sigma_{\varepsilon} \mathbf{V}^{\mathrm{T}}
$$

where $\Sigma_{\varepsilon}$ is a diagonal matrix in which the diagonal entries are equal to $\varepsilon$. It then can be proved that the new condition number of $\widetilde{\mathbf{C}}$ is $\left(\sigma_{\max }+\varepsilon\right) /\left(\sigma_{1}+\varepsilon\right) \ll \bar{C}$ when $\varepsilon$ is properly chosen. When the value of $\varepsilon$ is bigger than the smallest singular value of matrix $\mathbf{C}, \sigma_{1}$, but much smaller than the maximum singular value of matrix $\mathbf{C}, \sigma_{\max }$, the condition number will be dramatically improved. However, it should be mentioned here that $\varepsilon$ should not be very big, otherwise, it will distort the system too much. Mathematically speaking, such an operation will replace the original eigenproblem, $\mathbf{A}(k) \mathbf{t}=\mathfrak{R} \tilde{\mathbf{A}}(k) \mathfrak{R}^{\mathrm{T}} \mathbf{t}=0$, with a distorted problem, $\hat{\mathbf{A}}(k) \mathbf{t}=\mathfrak{R}\{\tilde{\mathbf{A}}(k)+\varepsilon \mathbf{I}\} \mathfrak{R}^{\mathrm{T}} \mathbf{t}=0$. Although the condition number now is improved, let us see what happens to the high order mode contamination now. Since $\varepsilon$ is added into the singular value, we now have $\mathbf{J}_{m}^{2}(k \rho)+\varepsilon \approx \varepsilon$ if $\varepsilon$ is much bigger than $\mathbf{J}_{m}^{2}(k \rho)$ for the high order mode under a small $k \rho$ (i.e., $m$ is big). However, for values near the true eigenvalue at low wave numbers (low order mode, i.e., $m$ is small), we have $\mathbf{J}_{m}^{2}(k \rho)+\varepsilon \approx \mathbf{J}_{m}^{2}(k \rho)$. 
Following the idea presented in section 4, we can choose an auxiliary system, whose boundary condition is linearly independent of that of the original problem, and then use the generalized singular-value decomposition method. Let us choose as our auxiliary system the Neumann case, i.e., $t=0$ on the boundary. Similar analytical derivation shows that the imaginary UT equation yields the eigenequation $\mathbf{J}_{m}^{\prime}(k \rho) \mathbf{J}_{m}(k \rho)=0$. If the Tikhonov's regularization method is also adopted, the singular value becomes $\mathbf{J}_{m}^{\prime}(k \rho) \mathbf{J}_{m}(k \rho)+\varepsilon^{*}$, where $\varepsilon^{*}$ is another small value designated for the $\mathbf{T}_{I}$ kernel. For the high order mode with a small argument $k \rho$, this value is very close to $\varepsilon^{*}$. When these two regularized matrices are treated together, the high mode contamination can be viewed as a "contaminated" spurious eigensolution since both $\mathbf{J}_{m}^{2}(k \rho)+\varepsilon \approx \varepsilon$ and $\mathbf{J}_{m}^{\prime}(k \rho) \mathbf{J}_{m}(k \rho)+\varepsilon^{*} \approx \varepsilon^{*}$ are small. The generalized singular-value decomposition method can then be adopted to filter them out. As mentioned in section 4, this method will treat rank deficiencies occurring simultaneously in the original and auxiliary system as the spurious eigensolution. Thus, this method is also available in eliminating the high order mode contamination in the regular BEM formulations if we take the high order mode contamination as a kind of spurious eigenvalue.

From the above discussion, it seems that the regular formulation has no difficulty dealing with eigenproblems. The above analytical derivation tells us that the basis functions we have in the imaginary UT equation or LM equation is nothing but $\mathbf{J}_{m}(k \rho)$ and $\mathbf{J}_{m}^{\prime}(k \rho)$. If there is a problem which has a solution needed to be represented in terms of $\mathrm{Y}_{m}(k \rho)$, can our method work? Also, since the basis functions we have now are $\mathbf{J}_{m}(k \rho)$ and $\mathbf{J}_{m}^{\prime}(k \rho)$ which are evidently non-singular functions, can we represent a singular field quantity, e.g., the displacement field of a membrane with a stringer? The answer is no; that is, we cannot expect to use the regular formulation to represent a field quantity having a singular behavior. To illustrate this, let us examine an annular region with an outer radius equal to $r_{1}$ and inner radius equal to $r_{2}$, and where the Dirichlet boundary condition is selected on the boundary as shown in Figure 5. The analytical eigenequation for this case was derived in reference [41], and is written as $\mathrm{J}_{m}\left(k r_{2}\right) \mathrm{Y}_{m}\left(k r_{1}\right)-\mathrm{J}_{m}\left(k r_{1}\right) \mathrm{Y}_{m}\left(k r_{2}\right)=0$. It can be seen for some special selections of $r_{1}$ and $r_{2}$ we have $\mathrm{Y}_{m}\left(k r_{1}\right)=0$ and $\mathrm{Y}_{m}\left(k r_{2}\right)=0$ at the same $k$ value; thus, we have a mode shape $\mathrm{Y}_{m}(k \tau)$ which is singular at $\tau=0$. Can we derive the same eigenequation from the imaginary UT equation? The analytical derivation is given as follows.

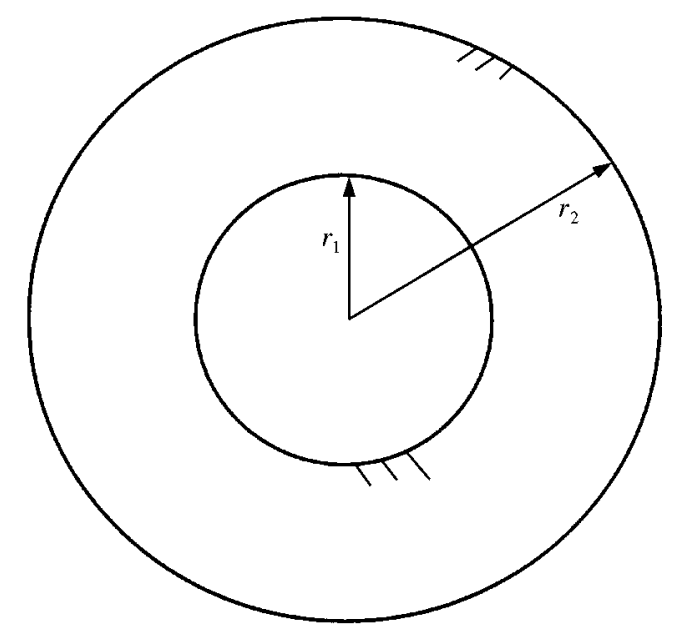

Figure 5. Illustration of an annular region. 
Assume that the outer radius and inner radius boundaries are all discretized into $2 \mathrm{~N}$ equal length elements, the imaginary UT formulation leads to

$$
\left[\begin{array}{ll}
\mathbf{A} & \mathbf{B} \\
\mathbf{D} & \mathbf{E}
\end{array}\right]\left[\begin{array}{l}
\mathbf{t}_{1} \\
\mathbf{t}_{2}
\end{array}\right]=\left[\begin{array}{l}
\mathbf{0} \\
\mathbf{0}
\end{array}\right],
$$

where $\mathbf{A}, \mathbf{B}, \mathbf{D}$ and $\mathbf{E}$ are influence matrices in circulant form, and $\mathbf{t}_{1}$ and $\mathbf{t}_{2}$ are flux values on the outer boundary with radius $r_{1}$ and inner boundary with radius $r_{2}$. Omitting lengthy derivation, we finally have the determinant value of leading coefficient matrix:

$$
\operatorname{det}\left[\begin{array}{ll}
\mathbf{A} & \mathbf{B} \\
\mathbf{D} & \mathbf{E}
\end{array}\right]=\mu_{0} \mu_{1} \cdots \mu_{2 N-1}=\mu_{0} \mu_{N} \prod_{m=1}^{N-1}\left(\mu_{m}\right)^{2},
$$

where

$$
\begin{gathered}
\mu_{m}=(2 \pi)^{2} r_{1} r_{2} \mathbf{J}_{m}\left(k r_{1}\right) \mathbf{J}_{m}\left(k r_{2}\right) \operatorname{det}\left|\begin{array}{cc}
\mathbf{J}_{m}\left(k r_{1}\right) & \mathbf{J}_{m}\left(k r_{2}\right) \\
\mathbf{J}_{m}\left(k r_{1}\right) & -\mathbf{J}_{m}\left(k r_{2}\right)
\end{array}\right|=0, \\
\mu_{2 N-m}=\mu_{m}, \quad m=0,1,2, \ldots, N .
\end{gathered}
$$

It should be mentioned here that each $\mu_{m}$ value in equation (37) actually represents the multiplication of two eigenconstraint equations; therefore, there are in total $4 \mathrm{~N}$ eigenconstraint equations in equation (36) rather than $2 \mathrm{~N}$ equations.

Based on the above, the imaginary UT equation leads to an incorrect conclusion that for every $k$ value, the system degenerates.

From the spectrum theory, we know that for a specific operator and a specific boundary condition, there exists a unique spectrum. If we miss some basis in the spectrum, no surprising that we can only represent solution of some problems but not for all problems. As for the regular BEM formulations, we can conclude that they are only suitable for problems with non-singular eigensolutions, not for those with singular solutions. For problems that cannot be solved using the regular BEM formulations, we call this ill-conditioned behavior the non-existence of solution due to an incomplete set of basis functions for representation.

\subsection{PLANE-WAVE FORMULATION}

In the following, the plane-wave formulation is considered. Again, a circular domain with a radius of $\rho$ is considered and the boundary condition is of Dirichlet type. $2 N$ equal length elements are used to discretize the circular boundary and $2 N$ plane waves are used as the complementary solutions, $P(\mathbf{x}, \phi)$, in equation (11). Omitting lengthy derivation, one can obtain

$$
\mathbf{G t}=\mathbf{0},
$$

with $\mathbf{G}$ being a circulant matrix as

$$
\mathbf{G}=\left[\begin{array}{ccccc}
g_{0} & g_{1} & g_{2} & \cdots & g_{2 N-1} \\
g_{2 N-1} & g_{0} & g_{1} & \cdots & g_{2 N-2} \\
g_{2 N-2} & g_{2 N-1} & g_{0} & \cdots & g_{2 N-3} \\
\vdots & \vdots & \vdots & \ddots & \vdots \\
g_{1} & g_{2} & g_{3} & \cdots & g_{0}
\end{array}\right]
$$


The entries in equation (39) can be expressed as

$$
g_{m}=\mathrm{e}^{\mathrm{i} k \rho \cos (m \Delta \psi)} \rho \Delta \psi,
$$

where $\Delta \psi \equiv 2 \pi / 2 N$ and $m \Delta \psi$ is the relative angle between the normal direction of a specific element and propagating direction of a specific plane wave. The $m$ th eigenvectors of the matrix $\mathbf{G}$ can be expressed as

$$
\left[\begin{array}{lllll}
1 & \gamma_{m} & \gamma_{m}^{2} & \cdots & \gamma_{m}^{2 N-1}
\end{array}\right]^{\mathbf{T}}
$$

where $\gamma_{m}=\mathrm{e}^{\mathrm{i} m \Delta \psi}$. The $m$ th eigenvalue, $\lambda_{m}$, then can be found as

$$
\lambda_{m}=\sum_{n=0}^{2 N-1} \gamma_{m}^{n} g_{n}=\sum_{n=0}^{2 N-1} \mathrm{e}^{\mathrm{i} n m \Delta \psi} g_{n} .
$$

By using that

$$
\mathrm{e}^{\mathrm{i} k \rho \cos \psi}=\sum_{l=-\infty}^{\infty}(i)^{l} \mathrm{~J}_{l}(k \rho) \mathrm{e}^{\mathrm{i} l \psi}
$$

and assigning $N$ to a big value, equation (42) can be rewritten as

$$
\begin{aligned}
\lambda_{m} & \cong \int_{0}^{2 \pi} \sum_{l=-\infty}^{\infty}(i)^{l} \mathrm{~J}_{l}(k \rho) \mathrm{e}^{\mathrm{i} l \psi} \cos (m \psi) \rho \mathrm{d} \psi \\
& =\pi \rho\left[(\mathrm{i})^{m} \mathbf{J}_{m}(k \rho)+(\mathrm{i})^{-m} \mathbf{J}_{-m}(k \rho)\right] \\
& =\pi \rho \mathbf{J}_{m}(k \rho)\left[i^{m}+(-\mathrm{i})^{-m}\right] .
\end{aligned}
$$

It then can be concluded that for a definite $m$, we have

$$
\lambda_{m}=\mathrm{i}^{m} 2 \pi \rho \mathbf{J}_{m}(k \rho) .
$$

After finding the eigenvalues of matrix $\mathbf{G}$, the determinant of matrix $\mathbf{G}$ can be obtained as

$$
\operatorname{det}(\mathbf{G})=\lambda_{0} \lambda_{N} \prod_{m=1}^{N-1} \lambda_{m}^{2},
$$

in which the relation of $\lambda_{m}=\lambda_{2 N-m}$ is used.

Then, it can be concluded that the eigenequation of the plane-wave method is simply $\mathrm{J}_{m}(k \rho)=0$ which is correct. Similar derivation for the Neumann problem using the plane-wave method can obtain the eigenequation is $\mathbf{J}_{m}^{\prime}(k \rho)=0$. From the above derivation, it is found that the plane-wave method will not yield the spurious eigensolutions. Further, it can be said that the plane-wave method is mathematically equivalent to the Trefftz method in this case since they all have the same basis functions as shown in equation (43). Although the plane-wave method does not suffer from the spurious eigensolution, the numerical instability of solution still exists in such a formulation. It can be seen from equation (45) that for small argument $k \rho$ and big $m$, the value of the $m$ th eigenvalue will be near to zero, thus misleading us to believe that the rank deficiency of matrix $\mathbf{G}$ occurs. Except for the numerical instability, the plane-wave method also fails in dealing with a multiply connected domain for the same reason that we introduce in the imaginary part formulation. 


\section{NUMERICAL EXAMPLES}

Example 1. A unit circular domain with the Dirichlet boundary condition, $u=0$.

\subsection{REAL UT EQUATION}

Choosing the auxiliary system as the Neumann-type boundary condition, $t=0$, let us first examine Proposition 2 and check the rank deficiency by means of QR factorization. For simplicity, only the real UT equation is chosen to represent the singular incomplete BEM formulation. The direct search method is adopted for the eigenvalue searching. Although there exist many better methods such as the Newton-Raphson method, the steepest descent method and so on, the direct search method is used because we want to illustrate the full spectrum. It is shown in Figure 6 that at some specific wave number the

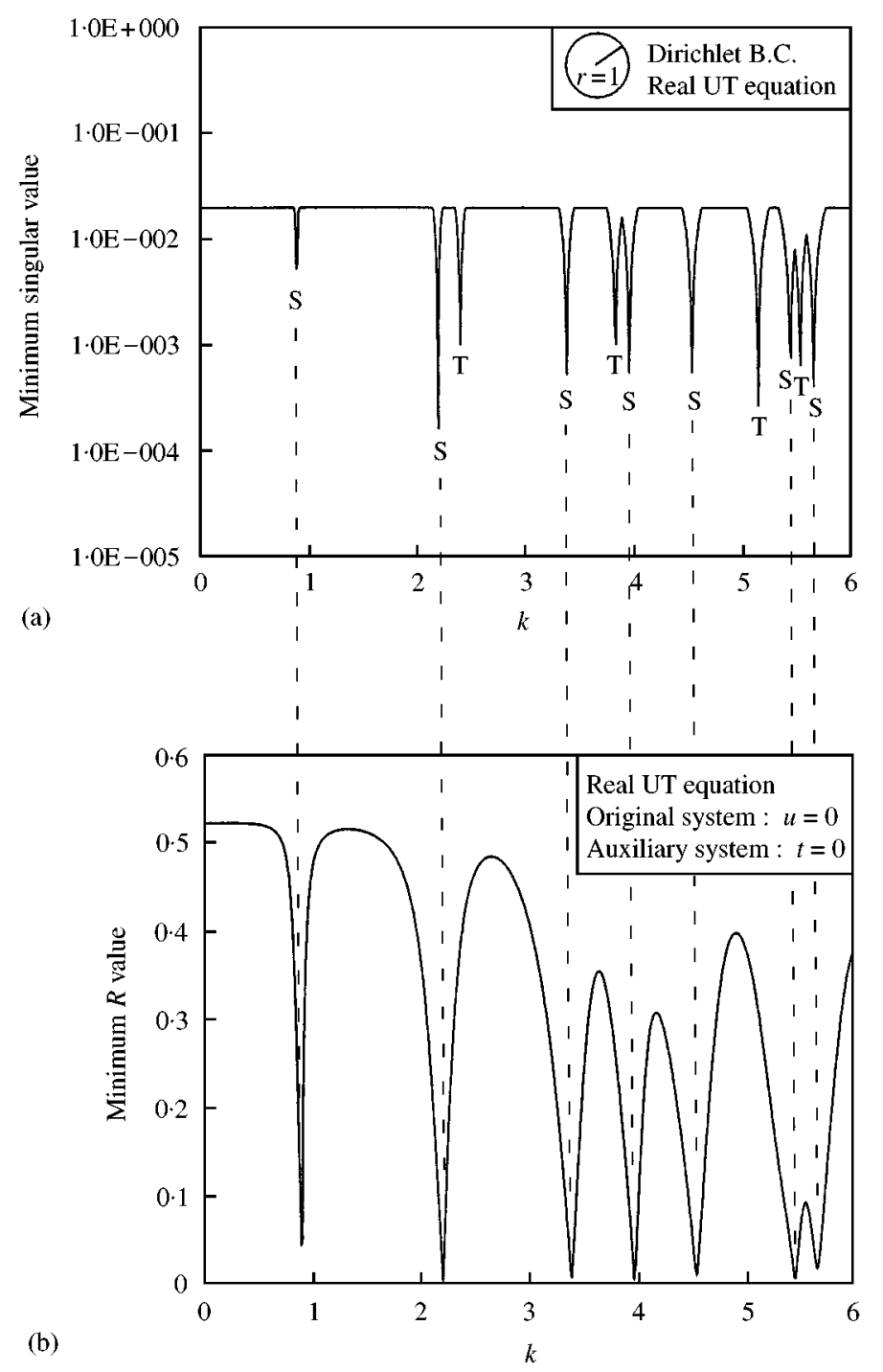

Figure 6. (a) Rank deficiency appearing in the system using the SVD method. (b) Spurious eigenvalues appearing in the system using QR factorization. S: Spurious eigenvalue; T: True eigenvalue. 
minimum value of the diagonal entries in matrix $\mathbf{R}$ (we will call it the minimum $R$ value in the remainder of this paper) can be very close to zero. Thus, such a wave number is recognized as the spurious eigenvalue. Comparing this figure with Figure 2(a), one can easily filter out the spurious eigenvalue. In Proposition 2, the auxiliary system can be arbitrary but needs to have homogeneous, linearly independent boundary conditions. To check this, another auxiliary system with the boundary condition given as $2 u+3 t=0$ is chosen. The minimum $R$ value for this combination is shown in Figure 7. One can find that our conclusion is correct. It then can be concluded that the spurious eigensolutions are common for all problems once the integral equation is selected. If only the spurious eigenvalue is in concern, one can simply take the QR factorization of $\left[\frac{U_{R}^{R}}{T_{R}^{R}}\right]$ for the real UT equation or $\left[\frac{L_{R}^{T}}{M_{R}}\right]$ for the real LM equation. The minimum $R$ value plot can then help us to find out spurious eigenvalues.

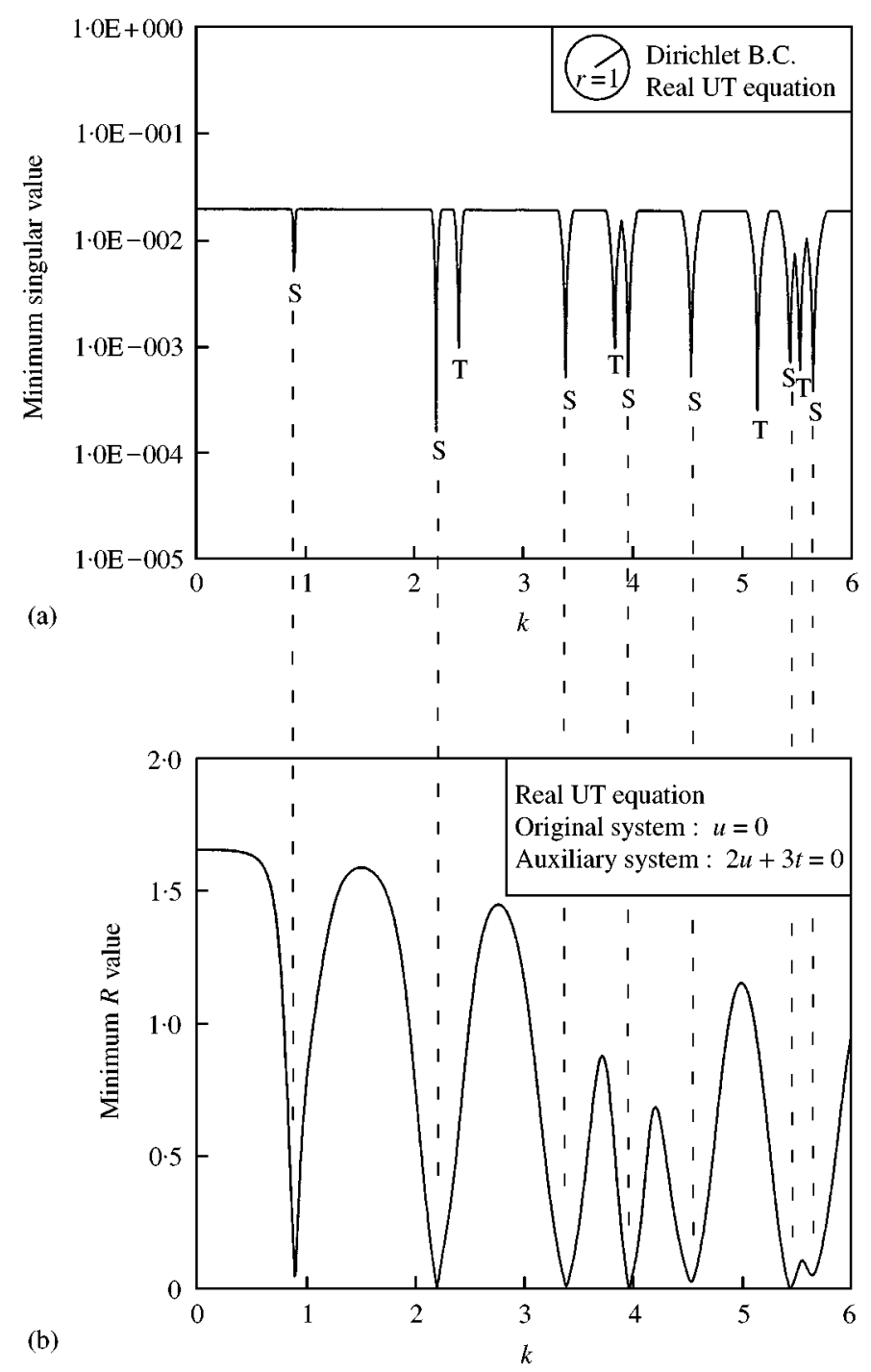

Figure 7. (a) Rank deficiency appearing in the system using the SVD method. (b) Spurious eigenvalues appearing in the system using QR factorization. S: Spurious eigenvalue; T: True eigenvalue. 


\subsection{IMAGINARY LM EQUATION}

The imaginary LM equation is chosen since it encounters the spurious eigenvalue problem and the numerical instability in this case, which is the worst one in the regular formulation. In this case, 60 elements were used along the boundary. When no treatment was adopted, the minimum singular-value plot had puzzling information as mentioned in the previous section and shown in Figure 4. After choosing an auxiliary system with $t=0$ on the boundary, we obtained the minimum generalized singular-value plot for the original system shown in Figure 8. In this figure and subsequent similar figures, the analytical values for eigenvalues are also illustrated by values inside the brackets. We find from this figure that the proposed method can successfully find true eigenvalues. Further, the first three eigenmodes are illustrated in Figures 9(a)-9(c). Remember that the method we propose here actually forces us to disturb the original system of equations a little bit; however, this seems to make little difference in the eigenmodes for the first three modes. Another auxiliary system with $2 u+3 t=0$ was selected, and the result for the generalized singular value of the original system is illustrated in Figure 10. From this figure, we can confirm that any system having homogeneous, linearly independent boundary conditions can be selected as an auxiliary system. We should mention here that for the complex-valued formulation and the real-valued formulation, the hypersingular integral equation requires much theoretical and numerical effort and, thus, is less welcome. However, in the imaginary BEM formulations, there is no difference between UT and LM equations since they are both regular formulations.

\subsection{THE PLANE-WAVE METHOD}

In this case, the plane-wave method proposed in section 2 was adopted. Since 60 elements were used to discretize the boundary, 60 plane-wave solutions with different $\phi$ angles were used to construct a sufficient number of constrained equations. The contamination phenomenon before the regularization did appear as shown in Figure 11. As mentioned in section 5, the plane-wave method will not result in spurious eigensolutions but still have the

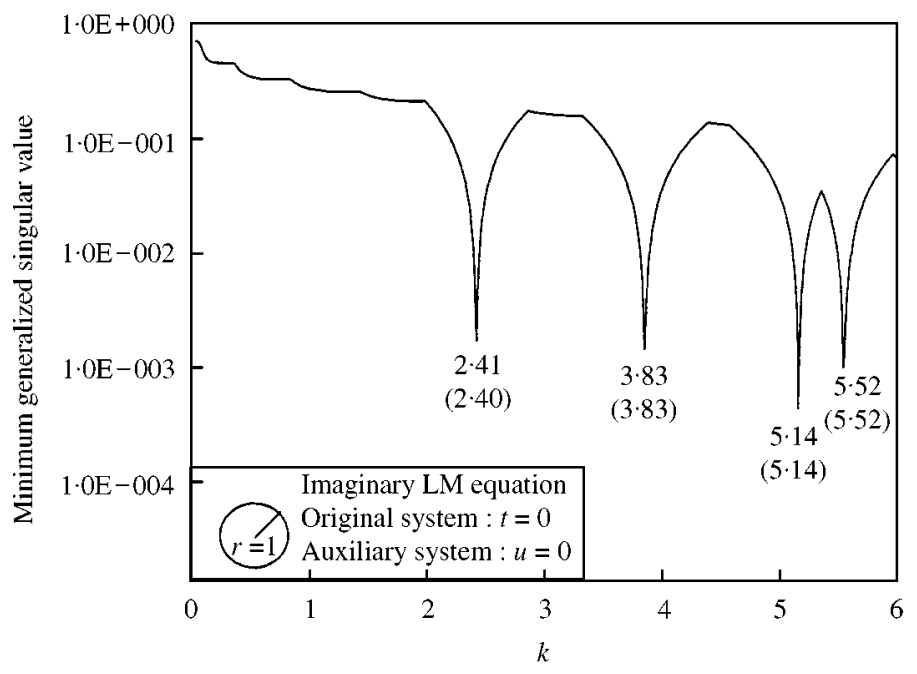

Figure 8. Eigenvalue searching for the Dirichlet problem using the imaginary LM equation. ( ): Analytical solution. 

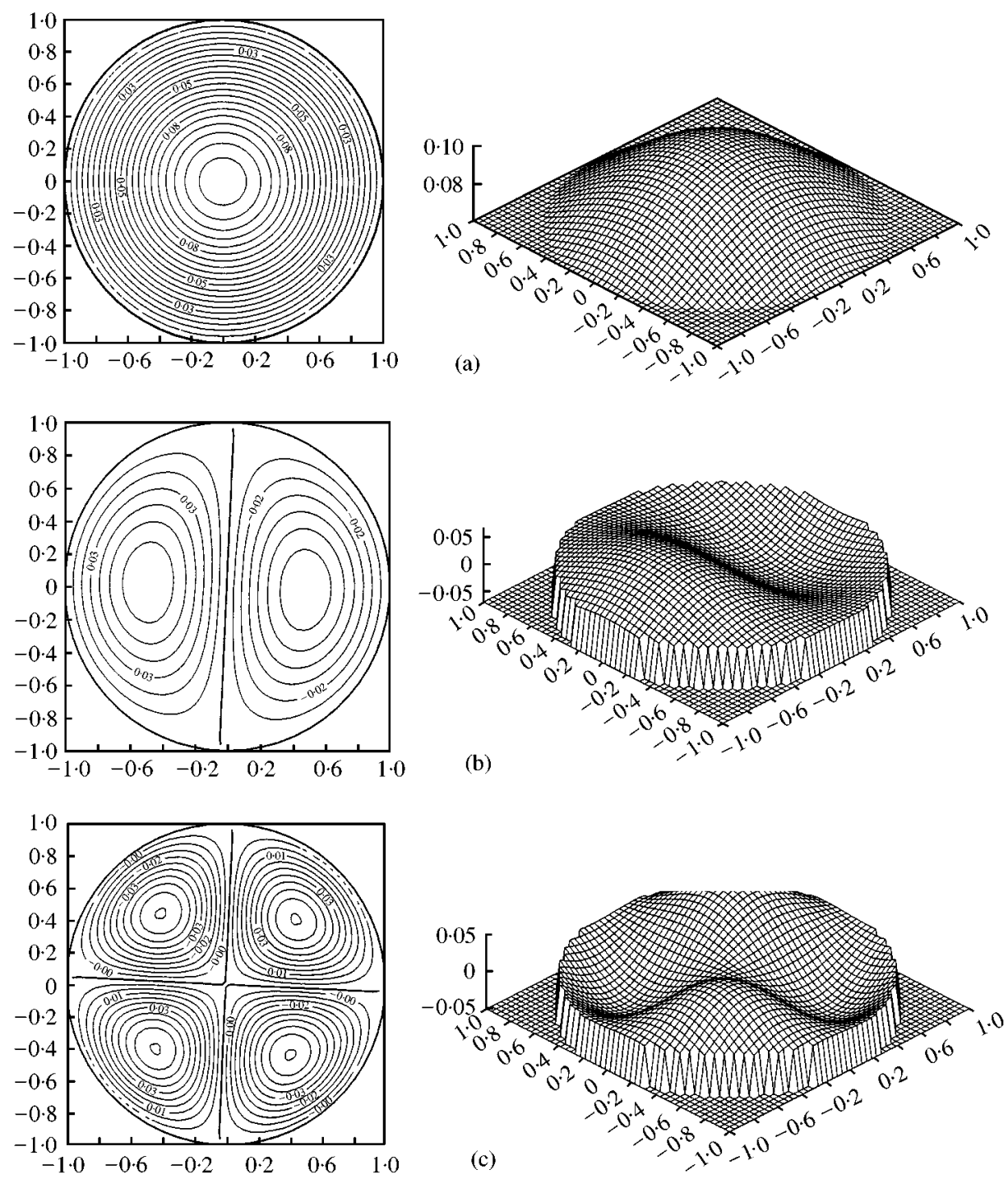

Figure 9. (a) The first mode shape of the Dirichlet case. (b) The second mode shape of the Dirichlet case. (c) The third mode shape of the Dirichlet case.

contamination phenomenon, i.e., the numerical instability. To deal with the numerical instability, the regularization technique should be employed. After using the regularization method with an auxiliary system having the boundary condition $t=0$, true eigenvalues could be determined easily as shown in Figure 12.

Example 2. A unit circular domain with the Neumann boundary condition, $t=0$.

\subsection{REAL UT EQUATION}

In this example, we use the generalized singular-value decomposition method mentioned in Proposition 1. The auxiliary system is the Dirichlet-type system. The minimum 


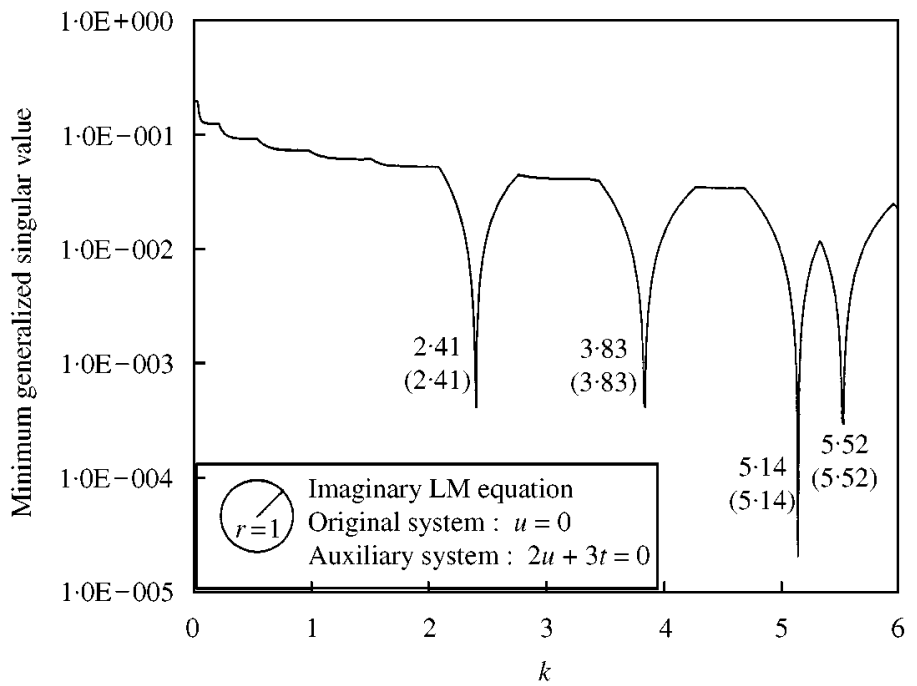

Figure 10. Eigenvalue searching for the Dirichlet problem using the imaginary LM equation with a different auxiliary system. ( ): Analytical solution.

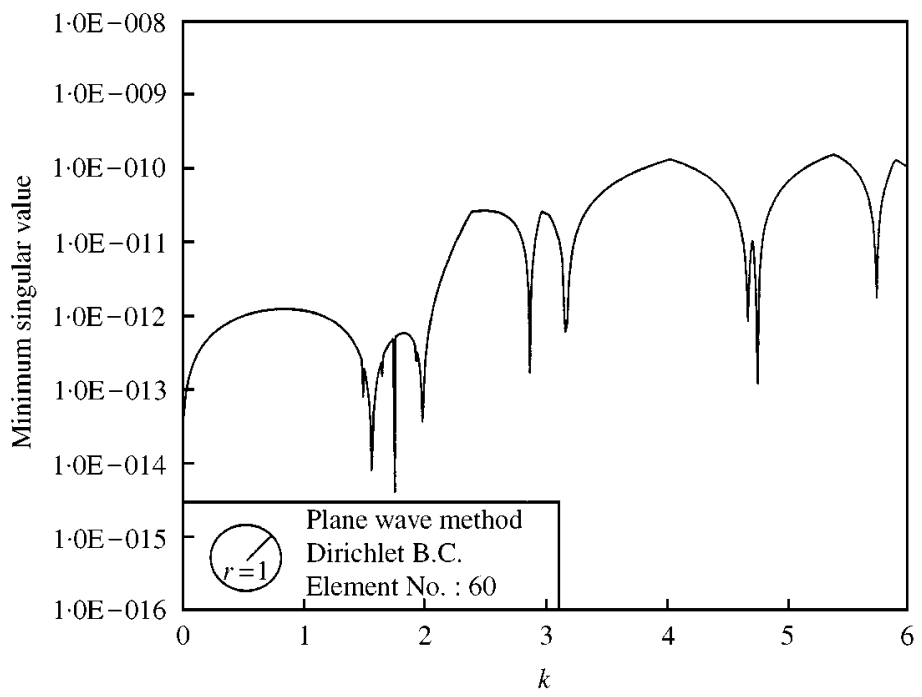

Figure 11. Contamination in eigenvalue searching using the plane-wave method.

generalized singular value versus wave number diagram is shown in Figure 13. It can be seen that one cannot distinguish spurious eigenvalues from true eigenvalues in this figure. The reason is explained as follows. We should note that at the spurious eigenvalue, one performs numerical calculation of an undetermined form of zero divided by zero while using the generalized singular-value decomposition. Theoretically speaking, the definite value of this undetermined form can be found by means of the L'Hospital rule. However, one may encounter difficulties in the numerical implementation. When the numerical methods one uses induce some errors, for example, collocation errors, mesh errors and so on, this 


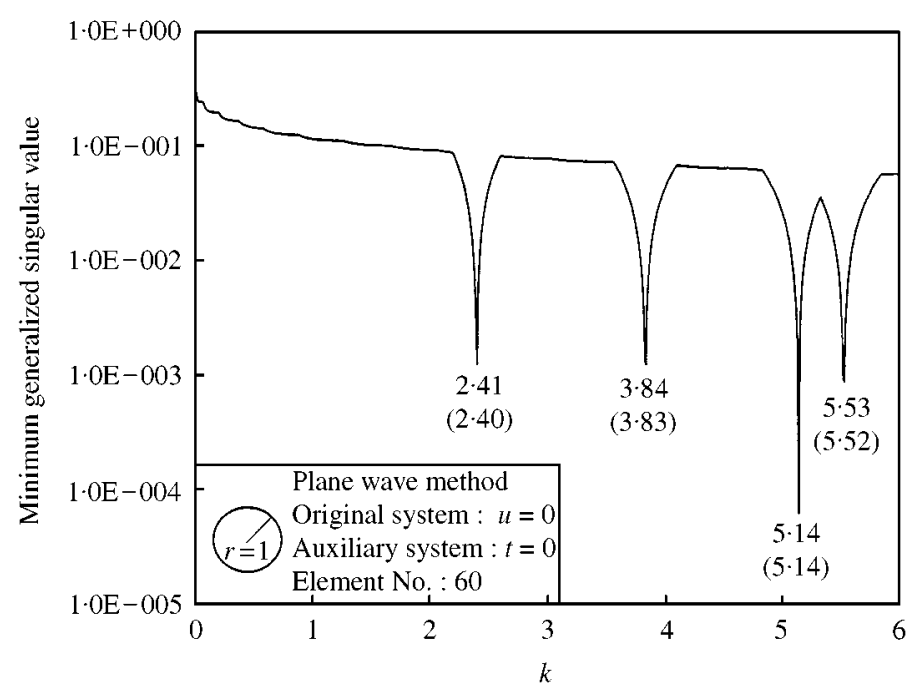

Figure 12. Eigenvalue searching for the Dirichlet problem using the plane-wave method. ( ): Analytical solution.

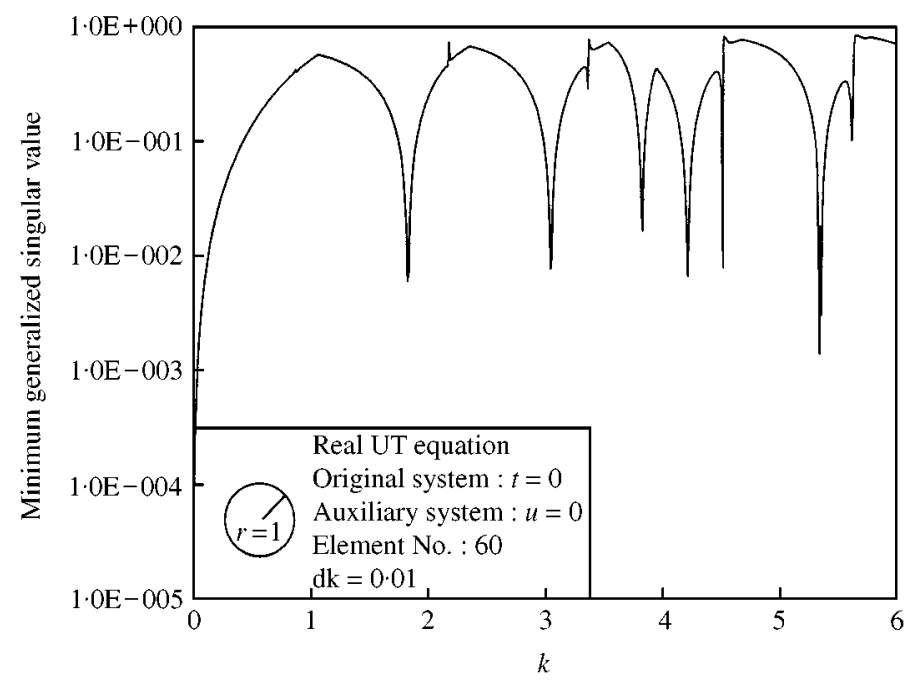

Figure 13. Numerical inaccuracy results in some spurious eigenvalues in the generalized singular value diagram.

eventually will cause failure for having rank deficiencies of both systems at the same wave number. Therefore, one may improve the numerical method in order to reduce errors such that good accuracy can be obtained; then, the chance of failure may be reduced. Since 60 elements are used in our analysis, using more elements possibly will still not prevent this phenomenon, and increasing number of elements implies that more degrees of freedom should be used, which is not economical. A natural guess is to choose for our auxiliary system a boundary condition that is a small perturbation from that of the original system. Then, one can expect that the true eigensolutions for both systems will be very close, and that the spurious one will occur at exactly the same position such that the generalized 
singular-value decomposition method can be implemented successfully. However, in order to find the true eigenvalue, one needs to make the searching step smaller. Therefore, we tried an auxiliary system having the boundary condition $t+0 \cdot 001 u=0$. The results are shown in Figure 14, where only true eigenvalues appear, thus verifying our conclusions.

The above method although can be successfully used to eliminate spurious eigenvalues, but we pay a price in the form of a finer searching step. Here, we propose another alternative that can eliminate spurious eigenvalues and save computation at the same time. If we go back and check the generalized singular-value decomposition method, we find that it actually combines two steps, QR factorization and C-S decomposition. Therefore, we can say that the wave number at which the minimum $R$ value approaches zero is the spurious one. However, to avoid possible failure in eliminating the rank deficiency at spurious eigenvalues due to numerical inaccuracy, one can set a threshold for the minimum $R$ value and omit calculation of generalized singular values when the minimum $R$ value is lower than the threshold. This method can be understood in the following way: since numerical implementation of zero divided by zero is not accurate enough for a rough calculation, one must simply give up on this attempt. However, if one connects points on the minimum generalized singular values versus wave number diagram by lines, no more drop down behaviors exist. Since this method combines the generalized singular-value decomposition method and the threshold, we call this method the threshold generalized singular-value decomposition method. Figure 15 shows the results obtained using this method. Although no more drop behaviors occur, kinks exist if the threshold is too small. Results obtained when a better threshold is used are shown in Figure 16. In this figure, kinks are less significant than those found in Figure 15. It must be mentioned here that the choice of the threshold value depends on the auxiliary system one selects. Generally speaking, if the boundary condition of the auxiliary system is very close to that of the original problem, it is then expected that the threshold value is smaller. On the contrary, the threshold value is bigger. From the minimum $R$ value plot, it is evidently the very position at which the numerical implementation of zero divided by zero will encounter difficulties is at the local minimum value. We propose to adopt a threshold value $\varepsilon$ in a way that while the wave number $k$ falls in the region of $\left\|k-k_{0}\right\| l_{c} \leqslant \varepsilon$, the numerical implementation ceases. In the

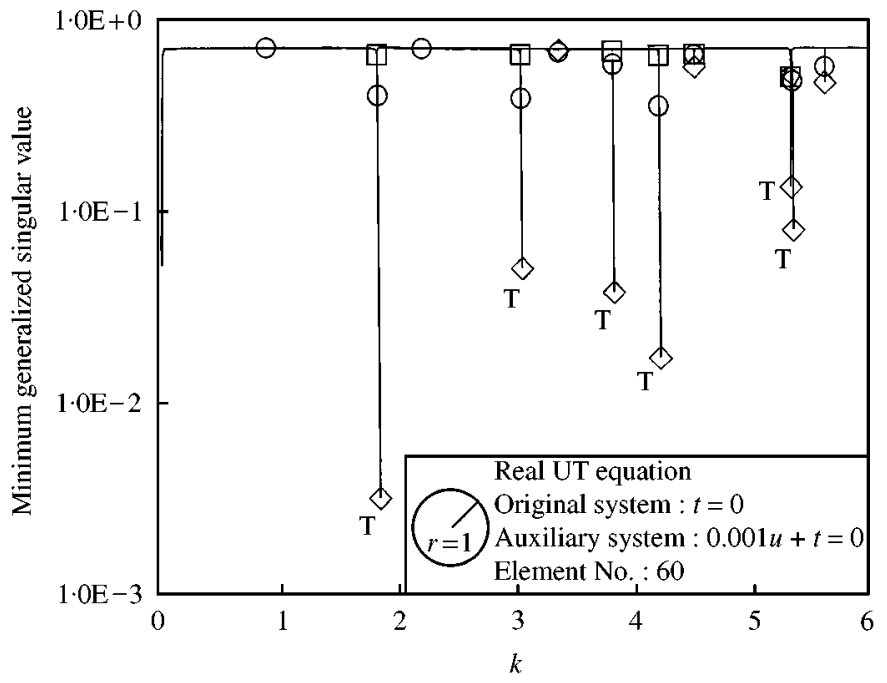

Figure 14. Elimination of numerical inaccuracy by selecting an auxiliary system very near the original one. $\square$, $\mathrm{dk}=0 \cdot 01 ; \bigcirc, \mathrm{dk}=0 \cdot 001 ; \diamond, \mathrm{dk}=0 \cdot 0001$. 


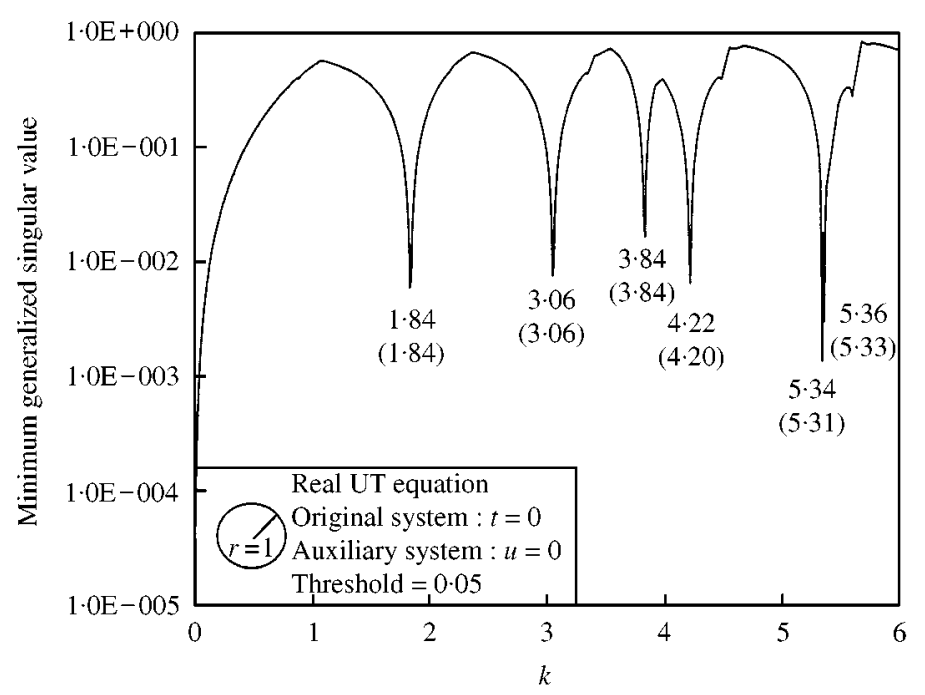

Figure 15. Kinks appearing in the threshold method near the spurious eigenvalues for a smaller threshold. ( ): Analytical solution.

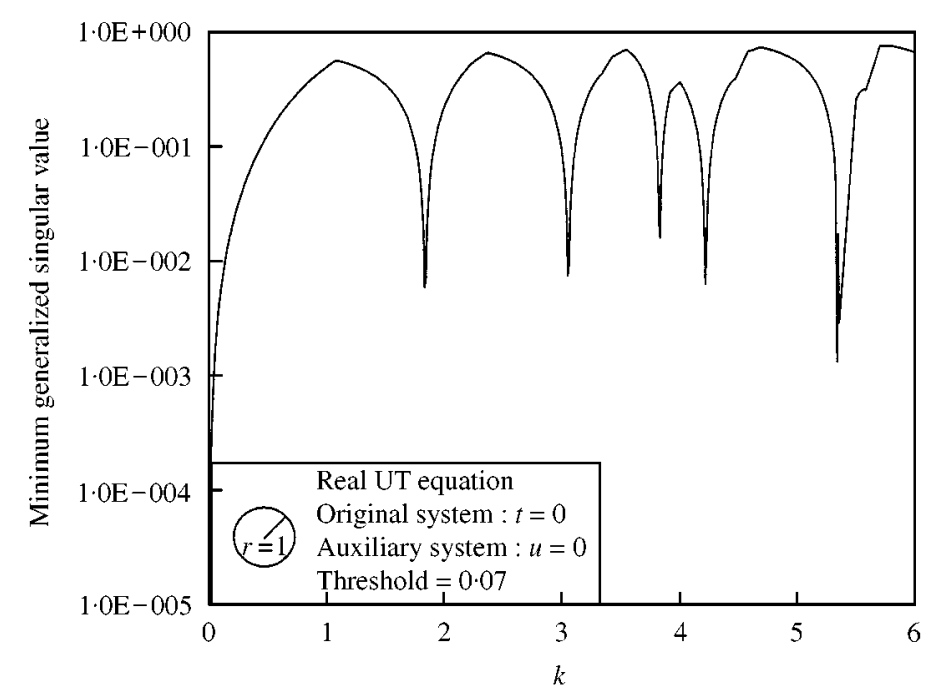

Figure 16. Smoothing of kinks by selecting a larger threshold.

above criterion, $k_{0}$ is the local minimum value in the minimum $R$ value plot, $l_{C}$ is the characteristic length for the domain interested and the largest distance between any two points on the boundary is selected as our characteristic length in the present study. For the cases we study, the threshold value is about $0 \cdot 01-0 \cdot 1$ according to numerical experiments.

\subsection{IMAGINARY UT EQUATION}

In this case, the imaginary UT equation has both the spurious eigensolutions and the numerical instability of solution. Sixty constant elements were used along the boundary. 


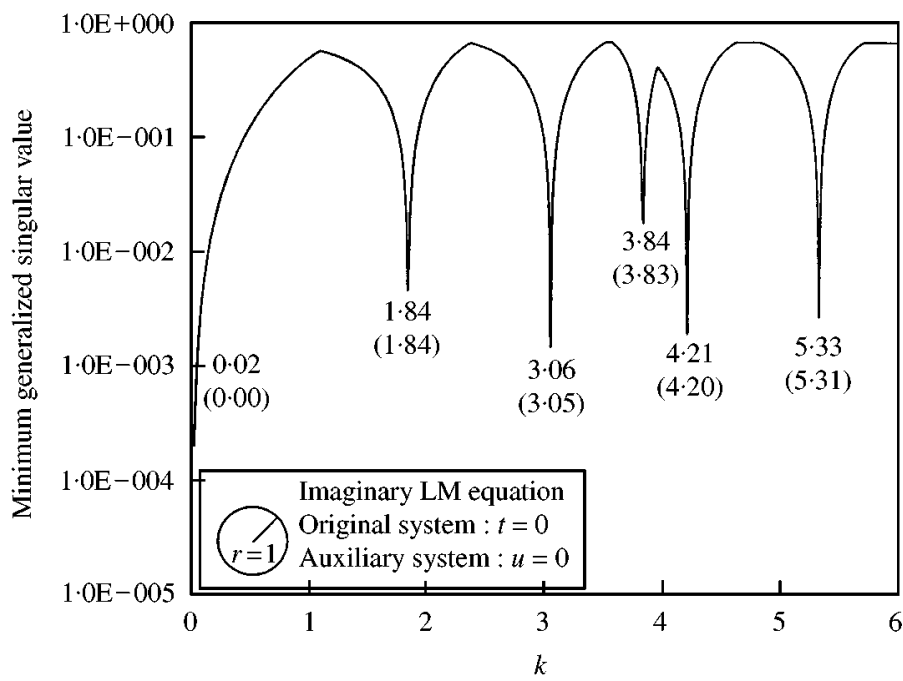

Figure 17. Eigenvalue searching for the Neumann problem using the imaginary UT equation. ( ): Analytical solution.

The result for the imaginary UT equation is illustrated in Figure 17. The selected auxiliary system had $u=0$ on the boundary. Basically, good results were obtained.

Example 3. A one by one square, Neumann problem, $t=0$.

\subsection{REAL LM EQUATION}

In this case, we solved the problem using the real LM equation. To check proposition 2, the minimum singular-value plot and the minimum $R$ value plot are shown in Figures 18(a) and 18 (b) respectively. From these figures, one can filter out the spurious eigenvalues. The results obtained using the threshold generalized singular-value decomposition method are shown in Figure 19.

\subsection{IMAGINARY UT EQUATION}

In section 5, it is seen that regular BEM formulations, no matter the imaginary formulations or the plane-wave method, can solve the problem of a circular domain. The purpose of this example is to show that the regular BEM method is still workable for cases without symmetry, i.e., cases with arbitrary shapes. It should be mentioned here that the imaginary UT or LM equation is not first proposed by us, these imaginary formulations have been studied previously by Chen et al. [24]. However, Chen et al. only illustrated their algorithm in an analytical sense by a circular domain. To implement the imaginary formulation in numerical sense, it is evidently difficult due to the numerical instability problem. Our method has a deeper insight comparing with previous studies since we point out the numerical instability problem existing in the regular formulation and further we proposed a regularization method. Since our method is independent of geometry, it can be qualified as a universal method. The numerical results are shown in Figure 20. It is not surprising that good results can be obtained by our proposed method. 

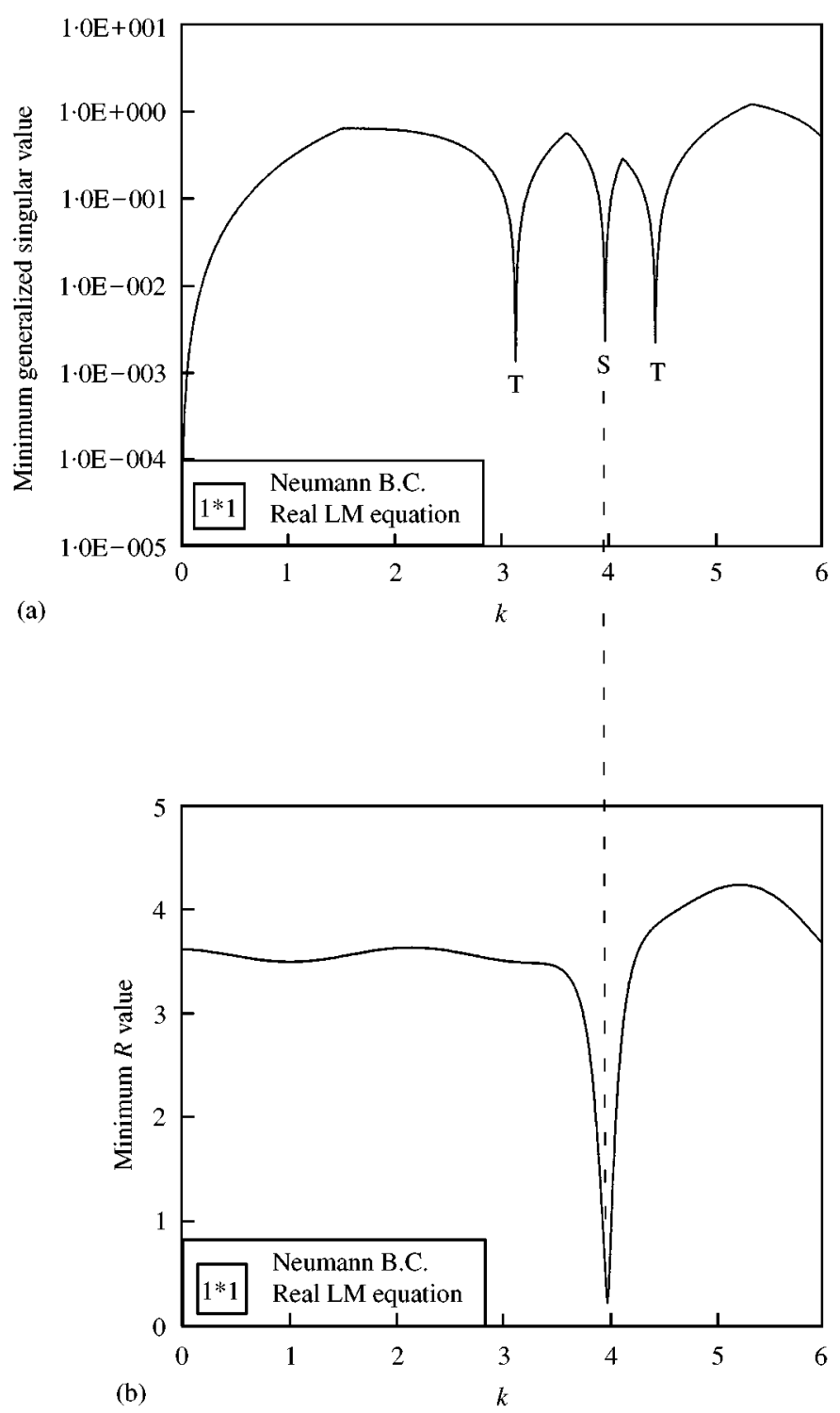

Figure 18. (a) Rank deficiency appearing in the system using the SVD method. (b) Spurious eigenvalues appearing in the system using QR factorization. S: Spurious eigenvalue; T: True eigenvalue.

\section{CONCLUDING REMARKS}

In this paper, incomplete BEM formulations for solving the Helmholtz equation have been examined. Due to the incompleteness of BEM formulations, one may encounter spurious eigensolutions, numerical instability of solution and nonexistence of solution. In this paper, the mathematical structure of spurious eigenvalues, which exists in the eigenvalue analysis using the real BEM formulation and imaginary BEM formulation, has been re-examined. It has been found that the spurious eigenvalues cause rank deficiency of the original system and the auxiliary system at the same time. Two alternative ways to filter out spurious eigenvalues have been proposed; one method uses the minimum singular-value 


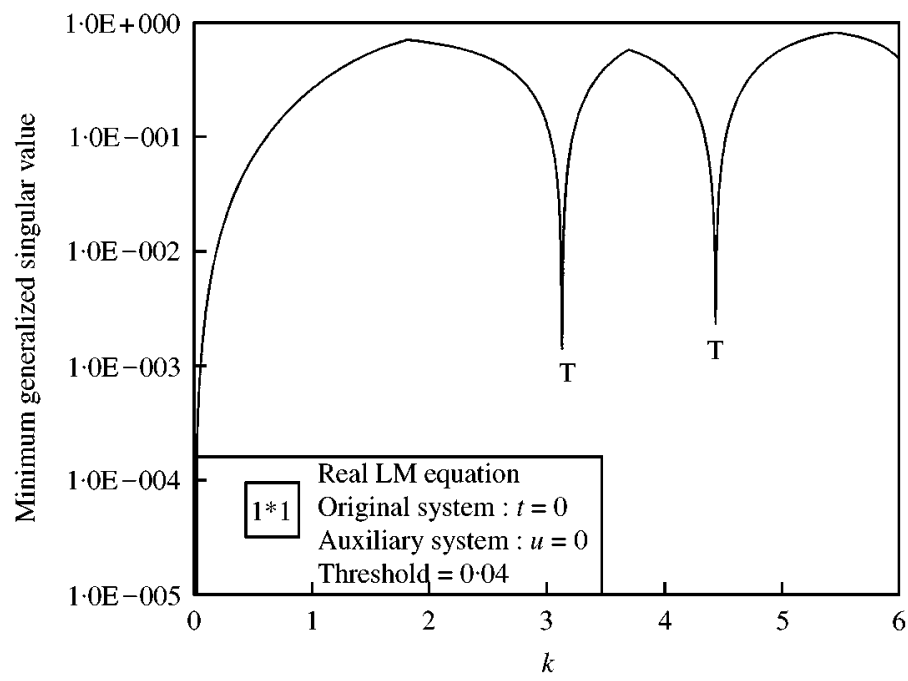

Figure 19. Filtering out spurious eigenvalues using the threshold method. T: True eigenvalue.

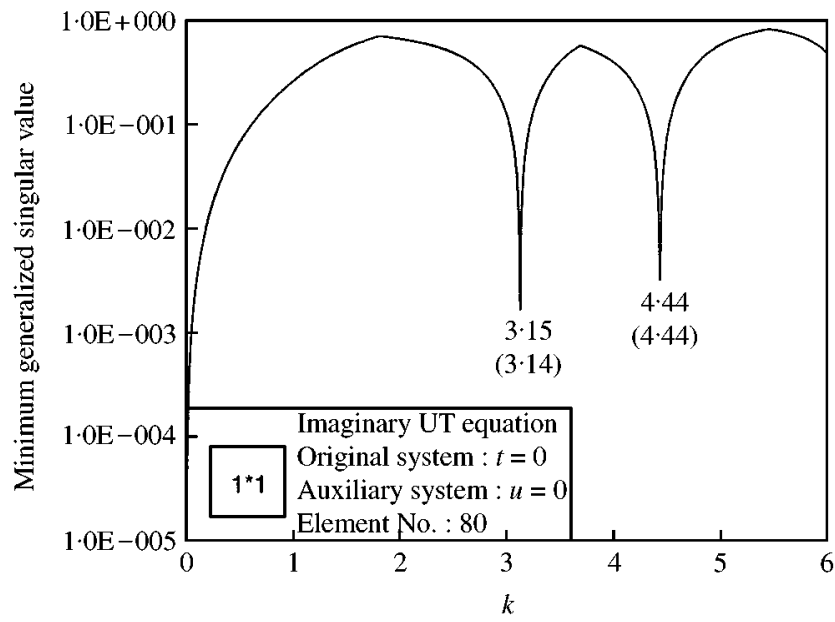

Figure 20. Eigenvalue searching for the Neumann case of a one by one square using the imaginary UT equation. ( ): Analytical solution.

plot of the original problem and the minimum $R$ value plot of $\mathrm{QR}$ factorization, and the other method uses the generalized singular-value decomposition method or the threshold generalized singular-value decomposition method. In addition, two regular BEM formulations for solving the eigenproblem have been proposed. It has been found that contamination exists in the regular BEM formulations. A unified method combining the Tikhonov's regularization technique and the generalized singular-value decomposition method has been proposed. Further, the possible non-existence of solution due to limited basis functions has been theoretically demonstrated. It has been concluded that the regular formulation cannot be used to search for a solution having singular behavior; thus, the regular formulation has its limitation. Despite this limitation, the regular BEM formulation has the advantage that it is easy to implement. 


\section{REFERENCES}

1. D. E. Beskos 1997 Applied Mechanics Reviews, ASME 50, 149-197. Boundary element method in dynamic analysis: Part II (1986-1996).

2. J. T. CHEN and F. C. Wong 1998 Journal of Sound and Vibration 217, 75-95. Dual formulation of multiple reciprocity method for the acoustic mode of a cavity with a thin partition.

3. I. Harari, P. E. Barbone, M. Slavutin and R. Shalom 1998 International Journal for Numerical Methods in Engineering 41, 1105-1131. Boundary infinite elements for the Helmholtz equation in exterior domains.

4. W. S. Hall and X. Q. MAO 1995 Engineering Analysis with Boundary Elements 16, 245-252. A boundary element investigation of irregular frequencies in electromagnetic scattering.

5. Y. Niwa, S. Kobayashi and M. Kitahara 1982 Development in Boundary Element Methods (P. K. BAnerJeE and R. Shaw, editors) Vol. 2. New York: Applied Science Publisher. Determination of eigenvalue by boundary element methods. Chapter 7.

6. D. E. BESKOS (editor). 1987 Boundary Element Methods in Mechanics. Amsterdam: North-Holland.

7. G. DE MEY 1977 International Journal for Numerical Methods in Engineering 11, 1340-1342. A simplified integral equation method for the calculation of the eigenvalues of Helmholtz equation.

8. J. R. Hutchinson 1985 Boundary Elements VII (C. A. BREBbia, G. MAIER, editors), 6.13-6.25 Berlin: Springer-Verlag. An alternative BEM formulation applied to membrane vibrations.

9. A. J. NowaK and C. A. BrebBia 1989 Computer Experiment in Fluid Flow (G. M. Calomagno and C. A. BrebBiA, editors), 265-270. Berlin: Comp. Mech. Pub./Springer. Solving Helmholtz equation by boundary elements using multiple reciprocity method.

10. N. KAMIYA and E. ANDOH 1993 Journal of Sound and Vibration 160, 279-287. Eigenvalue analysis by boundary element method.

11. N. KamiYa, E. ANDOH and K. Nogae 1993 Engineering Analysis with Boundary Elements 16, 203-207. Eigenvalue analysis by boundary element method: new developments.

12. C. F. NARDini and C. A. BrebBia 1983 Boundary Elements (C. A. BrebBia, T. FutAGAMI and M. TANAKA, editors), 719-730. Berlin: Springer. A new approach to free vibration analysis using boundary elements.

13. J. P. Agnantiaris, D. Polyzer and D. E. Beskos 1998 Computational Mechanics 21, 372-381. Three-dimensional structural vibration analysis by the dual reciprocity BEM.

14. M. A. Golberg, C. S. Chen, H. Bowman and H. Power 1998 Computational Mechanics 22, 61-69. Some comments on the use of radial basis functions in the dual reciprocity method.

15. N. KamiYa, E. ANDOH and K. NogaE 1996 Advances in Engineering Software 26, 219-227. A new complex-valued formulation and eigenvalue analysis of the Helmholtz equation by boundary element method.

16. W. YeIH, J. T. ChEn, K. H. CHEN and F. C. WONG 1998 Advances in Engineering Software 29, 1-6. A study on the multiple reciprocity method and complex-valued formulation for the Helmholtz equation.

17. J. T. CHEN and F. C. WONG 1997 Engineering Analysis with Boundary Elements 20, 25-33. Analytical derivations for one-dimensional eigenproblems using dual BEM and MRM.

18. D. Y. Liou, J. T. Chen and K. H. Chen 1999 Journal of the Chinese Institute of Civil and Hydraulic Engineering 11, 89-100. A new method for determining the acoustic modes of a two-dimensional sound field.

19. W. Yeih, J. T. Chen and C. M. Chang 1999 Engineering Analysis with Boundary Elements 23, 339-360. Applications of dual MRM for determining the natural frequencies and natural modes of an Euler-Bernoulli beam using the singular value decomposition method.

20. W. Yein, J. R. Chang, C. M. Chang and J. T. Chen 1999 Advances in Engineering Software 30, 459-468. Application of dual BEM for determining the natural frequencies and natural modes of a rod using the singular value decomposition method.

21. J. R. Chang, W. Yeih and J. T. Chen 1999 Computational Mechanics 24, 29-30. Determination of natural frequencies and natural modes of a rod using the dual BEM in conjunction with the domain partition technique.

22. Y. Y. Kim and J. H. Kang 1996 Journal of Acoustical Society of America 99, 2938-2946. Free vibration analysis of membranes using wave-type base functions.

23. S. W. KANG, J. M. LeE and Y. J. KANG 1999 Journal of Sound and Vibration 221, 117-132. Vibration analysis of arbitrarily shaped membranes using non-dimensional dynamic influence function. 
24. J. T. Chen, S. R. Kuo and K. H. Chen 1999 Journal of the Chinese Institute of Engineers 22, 729-739. A nonsingular integral formulation for the Helmholtz eigenproblems of a circular domain.

25. Y. K. Cheung, W. G. Jin and O. C. ZIEnkiewicz 1991 International Journal for Numerical Methods in Engineering 32, 63-78. Solution of Helmholtz equation by Trefftz Method.

26. W. G. Jin, Y. K. Cheung and O. C. ZIENKIEwICZ 1990 International Journal for Numerical Methods in Engineering 30, 1147-1161. Application of the Trefftz method in plane elasticity problems.

27. W. G. Jin, Y. K. Cheung and O. C. ZienkiewiCZ 1993 International Journal for Numerical Methods in Engineering 36, 765-781. Trefftz method for Kirchhoff plate bending problems.

28. J. JiROUSEK and A. WróBLEWSKI 1996 Archives of Computational Methods in Engineering 3, 323-434. T-elements: state of the art and future trends.

29. J. T. Chen, K. H. Chen and S. W. Chyuan 1999 Applied Acoustics 57, 293-325. Numerical experiments for acoustic modes of a square cavity using the dual boundary element method.

30. C. Patterson, J. L. Wearing and M. A. Sheikh 1986. Boundary Element Methods-Theory and Application, IOP Short Meeting Series 1, 57-95. A review of recent developments in boundary domain techniques.

31. Y. C. Wu 1999 Master Thesis, National Taiwan Ocean University, Keelung, Taiwan, R.O.C.. Applications of the generalized singular value decomposition method to the eigenproblem of Helmholtz equation.

32. C. X. HuAng 1999 Master Thesis, National Taiwan Ocean University, Keelung, Taiwan, R.O.C.. A study on true and spurious eigensolutions of two-dimensional acoustic cavities.

33. I. J. GooD 1968 Technometrices 11, 823-831. Some applications of the singular decomposition of a matrix.

34. V. C. Klema and A. J. Laub 1980 IEEE Transactions on Automatic Control, AC-25, 164-176. The singular decomposition: its computation and some applications.

35. J. T. CHEN 1998 Mechanics Research Communications 25, 529-534. On fictitious frequencies using dual series representation.

36. W. Benthi and A. SCHENCK 1997 Computers and Structures 65, 295-305. Nonexistence and nonuniqueness problems associated with integral equation methods in acoustics.

37. G. H. Golub and C. F. VAN LOAN 1989 Matrix Computations. Baltimore: The Johns Hopkins University Press, second edition.

38. J. T. Chen, S. R. KuO and C. X. HuAng 1999 IUT AM/I ACM/I ABEM Symposium on Advanced Mathematical Computational Mechanics, Aspects of Boundary Element Method, Cracow, Poland, 18-19. Analytical study and numerical experiments for true and spurious eigenvalues of a circular cavity using real part BEM.

39. S. R. KuO, J. T. Chen and C. X. HuAng 2000 International Journal for Numerical Methods in Engineering 48, 1401-1422. Analytical study and numerical experiments for true and spurious eigensolutions of a circular cavity using the real-part dual BEM.

40. A. N. TIKhonov 1963 Doklady Akademii Nauk, SSSR 151, 501-504. Solution of incorrectly formulated problems and regularization method.

41. J. R. Chang 1999 Ph.D. Thesis, National Taiwan Ocean University, Keelung, Taiwan, R.O.C.. Applications of domain partitioning on vibration problems using the dual BEM. 